\title{
Gene expression variability across cells and species shapes innate immunity
}

Tzachi Hagai ${ }^{1,2 *}$, Xi Chen ${ }^{1}$, Ricardo J. Miragaia ${ }^{1,3}$, Raghd Rostom ${ }^{1,2}$, Tomás Gomes ${ }^{1}$, Natalia Kunowska ${ }^{1}$, Johan Henriksson ${ }^{1}$, Jong-Eun Park ${ }^{1}$, Valentina Proserpio ${ }^{4,5}$, Giacomo Donati ${ }^{4,6}$, Lara Bossini-Castillo ${ }^{1}$, Felipe A. Vieira Braga ${ }^{1,7}$, Guy Naamati ${ }^{2}$, James Fletcher $^{8}$, Emily Stephenson ${ }^{8}$, Peter Vegh ${ }^{8}$, Gosia Trynka ${ }^{1}$, Ivanela Kondova ${ }^{9}$, Mike Dennis ${ }^{10}$, Muzlifah Haniffa ${ }^{8,11}$, Armita Nourmohammad ${ }^{12,13}$, Michael Lässig ${ }^{14}$ \& Sarah A. Teichmann ${ }^{1,2,15 *}$

\begin{abstract}
As the first line of defence against pathogens, cells mount an innate immune response, which varies widely from cell to cell. The response must be potent but carefully controlled to avoid self-damage. How these constraints have shaped the evolution of innate immunity remains poorly understood. Here we characterize the innate immune response's transcriptional divergence between species and variability in expression among cells. Using bulk and single-cell transcriptomics in fibroblasts and mononuclear phagocytes from different species, challenged with immune stimuli, we map the architecture of the innate immune response. Transcriptionally diverging genes, including those that encode cytokines and chemokines, vary across cells and have distinct promoter structures. Conversely, genes that are involved in the regulation of this response, such as those that encode transcription factors and kinases, are conserved between species and display low cell-to-cell variability in expression. We suggest that this expression pattern, which is observed across species and conditions, has evolved as a mechanism for fine-tuned regulation to achieve an effective but balanced response.
\end{abstract}

The innate immune response is a cell-intrinsic defence program that is rapidly upregulated upon infection in most cell types. It acts to inhibit pathogen replication while signalling the pathogen's presence to other cells. This programme involves the modulation of several cellular pathways, including production of antiviral and inflammatory cytokines, upregulation of genes that restrict pathogens, and induction of cell death $^{1,2}$.

An important characteristic of the innate immune response is the rapid evolution that many of its genes have undergone along the vertebrate lineage $e^{3,4}$. This rapid evolution is often attributed to pathogen-driven selection ${ }^{5-7}$.

Another hallmark of this response is its high level of heterogeneity among responding cells: there is extensive cell-to-cell variability in response to pathogen infection ${ }^{8,9}$ or to pathogen-associated molecular patterns (PAMPs) ${ }^{10,11}$. The functional importance of this variability is unclear.

These two characteristics-rapid divergence in the course of evolution and high cell-to-cell variability-seem to be at odds with the strong regulatory constraints imposed on the host immune response: the need to execute a well-coordinated and carefully balanced programme to avoid tissue damage and pathological immune conditions $^{12-15}$. How this tight regulation is maintained despite rapid evolutionary divergence and high cell-to-cell variability remains unclear, but it is central to our understanding of the innate immune response and its evolution.

Here, we study the evolution of this programme using two cells types-fibroblasts and mononuclear phagocytes-in different mammalian clades challenged with several immune stimuli (Fig. 1a).
Our main experimental system uses primary dermal fibroblasts, which are commonly used in immunological studies ${ }^{8,13}$. We compare the response of fibroblasts from primates (human and macaque) and rodents (mouse and rat) to polyinosinic:polycytidylic acid (poly(I:C)), a synthetic double-stranded RNA (dsRNA; Fig. 1a, left). Poly(I:C) is frequently used to mimic viral infection as it rapidly elicits an antiviral response ${ }^{16}$.

We comprehensively characterize the transcriptional changes between species and among individual cells in their innate immune response. We use population (bulk) transcriptomics to investigate transcriptional divergence between species, and single-cell transcriptomics to estimate cell-to-cell variability in gene expression. Using promoter sequence analyses along with chromatin immunoprecipitation with sequencing (ChIP-seq), we study how changes in the expression of each gene between species and across cells relate to the architecture of its promoter. Furthermore, we examine the relationship between cross-species divergence in gene coding sequence and expression and constraints imposed by host-pathogen interactions.

Additionally, we use a second system-bone marrow-derived mononuclear phagocytes from mouse, rat, rabbit and pig challenged with lipopolysaccharide (LPS), a commonly used PAMP of bacterial origin (Fig. 1a, right).

Together, these two systems provide insights into the architecture of the immune response across species, cell types and immune challenges.

\section{Transcriptional divergence in immune response}

First, we studied the transcriptional response of fibroblasts to stimulation with dsRNA (poly(I:C)) across the four species (human, macaque,

\footnotetext{
${ }^{1}$ Wellcome Sanger Institute, Cambridge, UK. ${ }^{2}$ EMBL- European Bioinformatics Institute, Cambridge, UK. ${ }^{3}$ Centre of Biological Engineering, University of Minho, Braga, Portugal. ${ }^{4}$ Department of Life Sciences and Systems Biology, University of Turin, Torino, Italy. ${ }^{5}$ Italian Institute for Genomic Medicine (IIGM), Torino, Italy. ${ }^{6}$ Molecular Biotechnology Center, University of Turin, Torino, Italy. ${ }^{7}$ Open Targets, Wellcome Sanger Institute, Cambridge, UK. ${ }^{8}$ Institute of Cellular Medicine, Newcastle University, Newcastle upon Tyne, UK. ${ }^{9}$ Division of Pathology and Microbiology, Animal Science Department, Biomedical Primate Research Centre, Rijswijk, The Netherlands. ${ }^{10}$ Research Department, Public Health England, National Infection Service, Porton Down, UK. ${ }^{11}$ Department of Dermatology and NIHR Newcastle Biomedical Research Centre, Newcastle Hospitals NHS Foundation Trust, Newcastle upon Tyne, UK. ${ }^{12}$ Max Planck Institute for Dynamics and Self-Organization, Göttingen, Germany. ${ }^{13}$ Department of Physics, University of Washington, Seattle, WA, USA. ${ }^{14}$ Institute for Biological Physics, University of Cologne, Cologne, Germany. ${ }^{15}$ Theory of Condensed Matter Group, Cavendish Laboratory, University of Cambridge, Cambridge, UK.*e-mail: tzachi@ebi.ac.uk; st9@sanger.ac.uk
} 
a

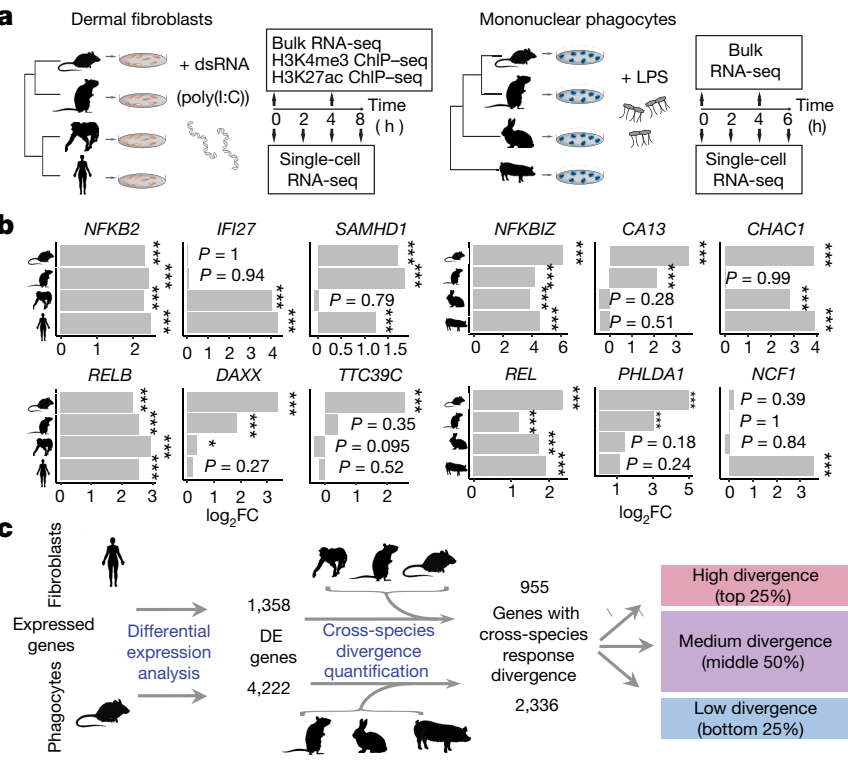

Fig. 1 Response divergence across species in innate immune response. a, Study design. Left, primary dermal fibroblasts from mouse, rat, human and macaque stimulated with dsRNA or controls. Samples were collected for bulk and single-cell RNA-seq and ChIP-seq. Right, primary bone marrow-derived mononuclear phagocytes from mouse, rat, rabbit and pig stimulated with LPS or controls. Samples were collected for bulk and single-cell RNA-seq. b, Left, fold-change (FC) in dsRNA stimulation in fibroblasts for sample genes across species (edgeR exact test, based on $n=6,5,3$ and 3 individuals from human, macaque, rat and mouse, respectively). Right, fold-change in LPS stimulation in phagocytes for sample genes across species (Wald test implemented in DESeq2, based on $n=3$ individuals from each species). False discovery rate (FDR)corrected $P$ values are shown $(* * * P<0.001, * * P<0.01, * P<0.05)$. c, Top, estimating each gene's level of cross-species divergence in transcriptional response to dsRNA stimulation in fibroblasts. Using differential expression analysis, fold-change in dsRNA response was assessed for each gene in each species. We identified 1,358 human genes as differentially expressed (DE) (FDR-corrected $q<0.01$ ), of which 955 had one-to-one orthologues across the four studied species. For each gene with one-toone orthologues across all species, a response divergence measure was estimated using: response divergence $=\log \left[1 / 4 \times \sum_{i, j}\left(\log \left[\mathrm{FC}\right.\right.\right.$ primate $\left._{i}\right]$ $\left.-[\log \mathrm{FC} \text { rodent } j]^{2}\right]$. Genes were grouped into low, medium and high divergence according to their response divergence values for subsequent analysis. Bottom, estimating each gene's level of cross-species divergence in LPS response in mononuclear phagocytes. A response divergence measure was estimated using: response divergence $=\log \left[1 / 3 \times \sum_{j}(\log [\mathrm{FC}\right.$ pig $]$ $-\log \left[\mathrm{FC}\right.$ glire $\left.\left.\left._{j}\right]\right)^{2}\right]$ (where glires are mouse, rat and rabbit).

rat and mouse). We generated bulk RNA-sequencing (RNA-seq) data for each species after $4 \mathrm{~h}$ of stimulation, along with respective controls (see Fig. 1a and Methods)

In all species, dsRNA treatment induced rapid upregulation of genes that encode expected antiviral and inflammatory products, including IFNB, TNF, IL1A and CCL5 (see also Supplementary Table 3). Focusing on one-to-one orthologues, we performed correlation analysis between species and observed a similar transcriptional response (Spearman correlation, $P<10^{-10}$ in all comparisons; Extended Data Fig. 1), as reported in other immune contexts ${ }^{17-19}$. Furthermore, the response tended to be more strongly correlated between closely related species than between more distantly related species, as in other expression programmes ${ }^{20-24}$

We characterized the differences in response to dsRNA between species for each gene, using these cross-species bulk transcriptomics data. While some genes, such as those encoding the NF- $\kappa B$ subunits RELB and NFKB2, respond similarly across species, other genes respond differently in the primate and rodent clades (Fig. 1b, left). For example, Ifi27 (which encodes a restriction factor against numerous viruses) is strongly upregulated in primates but not in rodents, whereas
Daxx (which encodes an antiviral transcriptional repressor) exhibits the opposite behaviour.

Similarly, in our second experimental system, which consists of lipopolysaccharide (LPS)-stimulated mononuclear phagocytes from mouse, rat, rabbit, and pig (Fig. 1b, right), some genes responded similarly across species (for example, $N f k b 2$ ), whereas others were highly upregulated only in specific clades (for example, Phlda1).

To quantify transcriptional divergence in immune responses between species, we focused on genes that were differentially expressed during the stimulation (see Methods). For simplicity, we refer to these genes as 'responsive genes' (Fig. 1c). In this analysis, we study the subset of these genes with one-to-one orthologues across the studied species. There are 955 such responsive genes in dsRNA-stimulated human fibroblasts and 2,336 in LPS-stimulated mouse phagocytes. We define a measure of response divergence by calculating the differences between the fold-change estimates while taking the phylogenetic relationship into account (Methods, Supplementary Figs. 1-7 and Supplementary Table 4).

For subsequent analyses, we split the 955 genes that were responsive in fibroblasts into three groups on the basis of their level of response divergence: (1) high-divergence dsRNA-responsive genes (the top $25 \%$ of genes with the highest divergence values in response to dsRNA across the four studied species); (2) low-divergence dsRNA-responsive genes (the bottom 25\%); and (3) genes with medium divergence across species (the middle $50 \%$; Fig. 1c). We performed an analogous procedure for the 2,336 LPS-responsive genes in phagocytes.

\section{Promoter architecture of diverging genes}

Next, we tested whether divergence in transcriptional responses is reflected in the conservation of promoter function and sequence. Using ChIP-seq, we profiled active histone marks in the fibroblasts of all species. The presence of trimethylation of lysine 4 on histone $\mathrm{H} 3$ (H3K4me3) in promoter regions of high-divergence genes was significantly less conserved between humans and rodents than was the presence of $\mathrm{H} 3 \mathrm{~K} 4 \mathrm{me} 3$ in promoters of low-divergence genes (Extended Data Fig. 2).

We then used the human H3K4me3 ChIP-seq peaks to define active promoter regions of the responsive genes in human fibroblasts. The density of transcription factor binding motifs (TFBMs) was significantly higher in the active promoter regions of high-divergence genes than in low-divergence genes (Fig. 2a). Notably, when comparing the conservation of the core promoter regions in high- versus lowdivergence dsRNA-responsive genes, we found that genes that diverge highly in response to dsRNA show higher sequence conservation in this region (Fig. 2b).

This unexpected discordance may be related to the fact that promoters of high- and low-divergence genes have distinctive architectures, associated with different constraints on promoter sequence evolution ${ }^{18,25,26}$. Notably, promoters containing TATA-box elements tend to have most of their regulatory elements in regions immediately upstream of the transcription start site (TSS). These promoters are thus expected to be more conserved. The opposite is true for $\mathrm{CpG}$ island $(\mathrm{CGI})^{26,27}$ promoters. Indeed, we found that TATA-boxes are associated with higher transcriptional divergence, while genes with CGIs diverge more slowly, both in fibroblasts and phagocytes (Fig. 2c; Extended Data Fig. 3). Thus, a promoter architecture enriched in TATA-boxes and depleted of CGIs is associated with higher transcriptional divergence, while entailing higher sequence conservation upstream of these genes $^{18,26,27}$.

\section{Transcriptional divergence of cytokines}

We next investigated whether different functional classes among responsive genes are characterized by varying levels of transcriptional divergence. To this end, we divided responsive genes into categories according to function (such as cytokines, transcriptional factors and kinases) or the processes in which they are known to be involved (such as apoptosis or inflammation). 
a
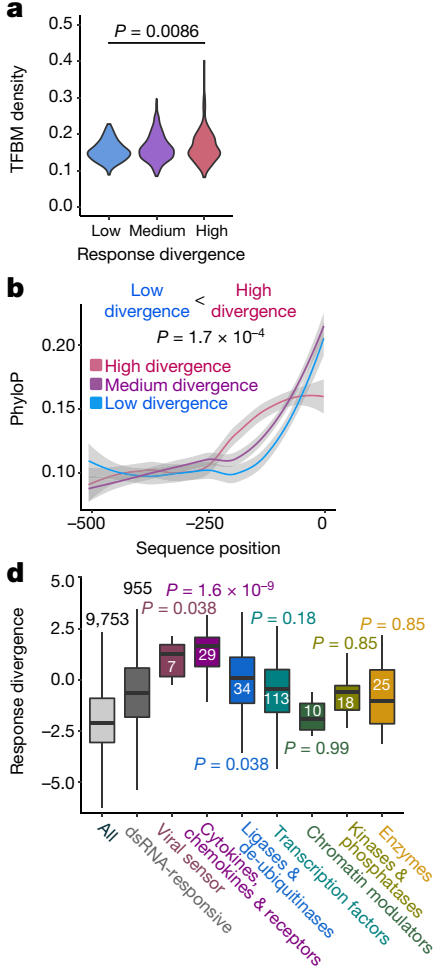
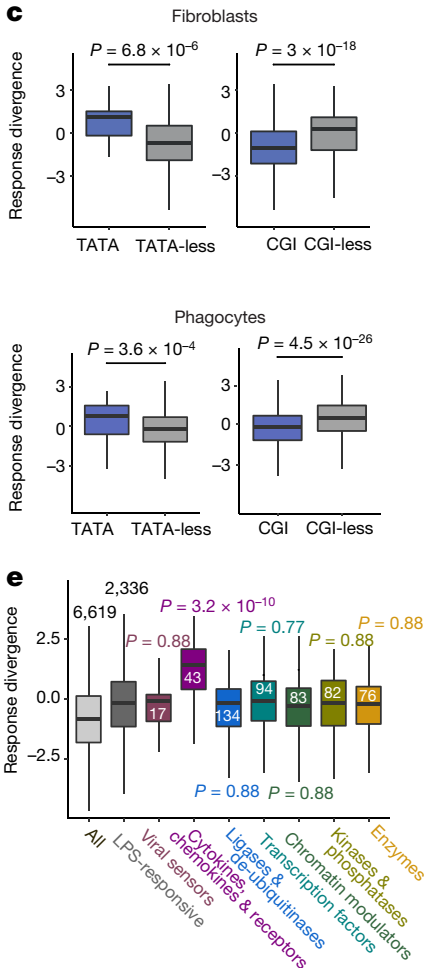

Fig. 2 Transcriptionally divergent genes have unique functions and promoter architectures. a, TFBM density in active promoters and response divergence. For each gene studied in fibroblast dsRNA stimulation, the total number of TFBM matches in its $\mathrm{H} 3 \mathrm{~K} 4 \mathrm{me} 3$ histone mark was divided by the length of the mark (human marks were used; $n=879$ differentially expressed genes with ChIP-seq data). Highdivergence genes have higher TFBM density than low-divergence genes (one-sided Mann-Whitney test). b, Promoter sequence conservation and response divergence in fibroblast dsRNA stimulation. Sequence conservation values are estimated with phyloP7 for 500 base pairs upstream of the transcription start site (TSS) of the human gene. Mean conservation values of each of the 500 base pairs upstream of the TSS are shown for high-, medium- and low-divergence genes ( $n=840$ genes). Genes that are highly divergent have higher sequence conservation (one-sided Kolmogorov-Smirnov test). The 95\% confidence interval for predictions from a linear model computed by geom_loess function is shown in grey. c, Comparison of divergence in response of genes with and without a TATA-box and a CGI in fibroblast dsRNA stimulation and phagocyte LPS stimulation. TATA-box matches and CGI overlaps were computed with respect to the TSS of human genes in fibroblasts ( $n=955$ genes), and to the TSS of mouse genes in phagocytes $(n=2,336)$.

d, Distributions of divergence values of 9,753 expressed genes in fibroblasts, 955 dsRNA-responsive genes and different functional subsets of the dsRNAresponsive genes (each subset is compared with the set of 955 genes using a one-sided Mann-Whitney test and FDR-corrected $P$ values are shown). e, Distributions of divergence values of 6,619 expressed genes in phagocytes, 2,336 LPS-responsive genes and different functional subsets of the LPSresponsive genes (each subset is compared with the set of 2,336 genes using a one-sided Mann-Whitney test and FDR-corrected $P$ values are shown). Violin plots show the kernel probability density of the data. Boxplots represent the median, first quartile and third quartile with lines extending to the furthest value within 1.5 of the interquartile range (IQR).

Genes related to cellular defence and inflammation-most notably cytokines, chemokines and their receptors (hereafter 'cytokines') tended to diverge in response significantly faster than genes involved in apoptosis or immune regulation (chromatin modulators, transcription factors, kinases and ligases) (Fig. 2d, e, Extended Data Fig. 4, Supplementary Fig. 1).

Cytokines also had a higher transcriptional range in response to immune challenge (a higher fold-change). Regressing the fold-change from the divergence estimates resulted in reduction of the relative

divergence of cytokines versus other responsive genes, but the difference still remained (Supplementary Fig. 2). Cytokine promoters are enriched in TATA-boxes (17\% versus $2.5 \%, P=1.1 \times 10^{-3}$, Fisher's exact test) and depleted of CGIs ( $14 \%$ versus $\left.69 \%, P=1.6 \times 10^{-9}\right)$, suggesting that this promoter architecture is associated both with greater differences between species (response divergence) and larger changes between conditions (transcriptional range).

\section{Cell-to-cell variability in immune response}

Previous studies have shown that the innate immune response displays high variability across responding cells ${ }^{28,29}$. However, the relationship between cell-to-cell transcriptional variability and response divergence between species is not well understood.

To study heterogeneity in gene expression across individual cells, we performed single-cell RNA-seq in all species in a time course following immune stimulation. We estimated cell-to-cell variability quantitatively using an established measure for variability: distance to median $(\mathrm{DM})^{30}$.

We found a clear trend in which genes that were highly divergent in response between species were also more variable in expression across individual cells within a species (Fig. 3a). The relationship between rapid divergence and high cell-to-cell variability held true in both the 955 dsRNA-responsive genes in fibroblasts and the 2,336 LPS-responsive genes in phagocytes. This can be observed across the stimulation time points and in different species (Extended Data Figs. 5, 6). We analysed in depth the relationship between transcriptional divergence and cell-to-cell variability by using additional immune stimulation protocols (Supplementary Figs. 8, 9), and different experimental and computational approaches (Extended Data Fig. 7, Supplementary Figs. 10-13). Notably, the trends we observed are not a result of technical biases due to low expression levels in either the bulk or the single-cell RNA-seq data (Supplementary Figs. 14, 15).

Next, we examined the relationship between the presence of promoter elements (CGIs and TATA-boxes) and a gene's cell-to-cell variability. Genes that are predicted to have a TATA-box in their promoter had higher transcriptional variability, whereas CGI-containing genes tended to have lower variability (Fig. 3b), in agreement with previous findings ${ }^{31}$. Thus, both transcriptional variability between cells (Fig. 3b) and transcriptional divergence between species (Fig. 2c) are associated with the presence of specific promoter elements.

\section{Transcriptional variability of cytokines}

We subsequently compared the response divergence across species with the transcriptional cell-to-cell variability of three groups of responsive genes with different functions: cytokines, transcription factors, and kinases and phosphatases (hereafter 'kinases'; Fig. 3c, Extended Data Fig. 8). In contrast to kinases and transcription factors, many cytokines display relatively high levels of cell-to-cell variability (Extended Data Fig. 9), being expressed only in a small subset of responding cells (Extended Data Fig. 10). This has previously been reported for several cytokines ${ }^{29}$. For example, IFNB is expressed in only a small fraction of cells infected with viruses or challenged with various stimuli ${ }^{8,11,32}$. Here, we find that cells show high levels of variability in expression of cytokines from several families (for example, IFNB, CXCL10 and CCL2).

Cell-to-cell variability of cytokines remains relatively high in comparison to kinases and transcription factors during a time course of 2, 4 and $8 \mathrm{~h}$ after dsRNA stimulation of fibroblasts (Extended Data Fig. 9). This pattern is similar across species, and can also be observed in LPS-stimulated phagocytes (Extended Data Fig. 9). Thus, the high variability of cytokines and their expression in a small fraction of stimulated cells across all time points is evolutionarily conserved.

Cytokines tended to be co-expressed in the same cells, raising the possibility that their expression is coordinated (see Supplementary Information and Supplementary Fig. 16). We also identified genes whose expression was correlated with cytokines in human fibroblasts and showed that their orthologues tend to be co-expressed with cytokines in other species. This set is enriched with genes known to be involved in cytokine regulation (Supplementary Table 5). 

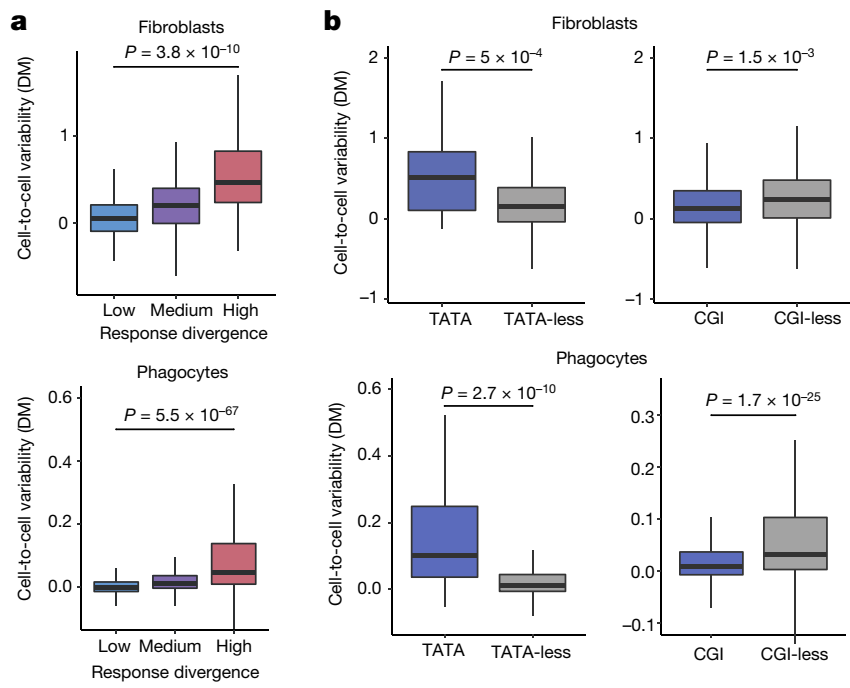

Phagocytes
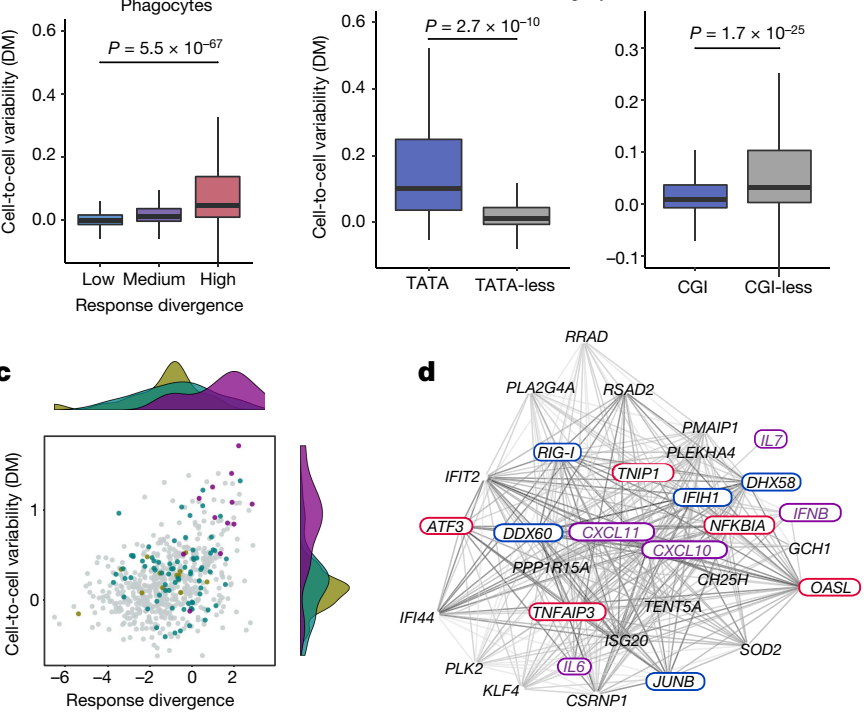

Fig. 3 Cell-to-cell variability in immune response corresponds to response divergence. a, Comparison of divergence in response across species with transcriptional variability between individual cells. Top, fibroblast dsRNA stimulation (variability measured in $n=55$ human cells, following $4 \mathrm{~h}$ dsRNA stimulation). Bottom, phagocyte LPS stimulation (variability measured in $n=3,293$ mouse cells, following $4 \mathrm{~h}$ LPS stimulation). Genes classified as high-, medium- or low-divergence according to level of response divergence. Cell-to-cell variability values of high-divergence genes were compared with those of low-divergence genes (one-sided Mann-Whitney test). b, Comparison of cell-to-cell variability of genes with and without a TATA-box and a CGI, in fibroblast dsRNA stimulation and phagocyte LPS stimulation (one-sided Mann-Whitney test). Cell-to-cell variability values are from DM estimations of human fibroblasts stimulated with dsRNA for $4 \mathrm{~h}$ ( $n=55$ cells) and from mouse phagocytes stimulated with LPS for $4 \mathrm{~h}$ ( $n=3,293$ cells). c, Scatter plot showing divergence in response to dsRNA in fibroblasts across species and transcriptional cell-to-cell variability in human cells following $4 \mathrm{~h}$ of dsRNA stimulation ( $n=684$ dsRNA-responsive genes). Purple, cytokines; green, transcription factors; beige, kinases. The distributions of divergence and variability values of these groups are shown above and to the right of the scatter plot, respectively. d, A network showing genes that correlate positively in expression with the chemokine gene CXCL10 across cells (Spearman correlation, $\rho>0.3$ ), in at least two species (one of which is human), following dsRNA treatment in fibroblasts (based on $n=146,74,175$ and 170 human, macaque, rat and mouse cells, respectively). Purple, cytokines; red, positive regulators of cytokine expression; blue, negative regulators. Colours of lines, from light to dark grey, reflect the number of species in which this pair of genes was correlated. Boxplots represent the median, first quartile and third quartile with lines extending to the furthest value within $1.5 \times \mathrm{IQR}$.

As an example, we focused on the genes whose expression is positively correlated with the chemokine CXCL10 in at least two species (Fig. 3d). This set includes four cytokines co-expressed with CXCL10 (in purple), as well as known positive regulators of the innate immune response and cytokine production (in blue), such as the viral sensors IFIH1 (also known as MDA5) and RIG-I (also known as DDX58) This is in agreement with previous evidence that IFNB expression is limited to cells in which important upstream regulators are expressed at sufficiently high levels ${ }^{8,11,32}$. Here, we show that this phenomenon of co-expression with upstream regulators applies to a wider set of cytokines and is conserved across species. Notably, cytokines were co-expressed not only with their positive regulators but also with genes that are known to act as negative regulators of cytokine expression or cytokine signalling (in red), suggesting that cytokine expression and function is tightly controlled at the level of individual cells.

\section{The evolutionary landscape of innate immunity}

Many immune genes, including several cytokines and their receptors, have been shown to evolve rapidly in coding sequence ${ }^{3,33}$. However, it is not known how divergence in coding sequence relates to transcriptional divergence in innate immune genes. Using the set of 955 dsRNA-responsive genes in fibroblasts, we assessed coding sequence evolution in the three subsets of low-, medium- and high-divergence genes (as defined in Fig. 1c).

We compared the rate at which genes evolved in their coding sequences with their response divergence by considering the ratio of non-synonymous (dN) to synonymous (dS) nucleotide substitutions. Genes that evolved rapidly in transcriptional response had higher coding sequence divergence (higher $\mathrm{dN} / \mathrm{dS}$ values) than dsRNAresponsive genes with low response divergence (Fig. $4 \mathrm{a}$ ).

Rapid gene duplication and gene loss have been observed in several important immune genes ${ }^{34-39}$ and are thought to be a result of pathogendriven pressure ${ }^{40,41}$. We therefore tested the relationship between a gene's divergence in response and the rate at which the gene's family has expanded and contracted in the course of vertebrate evolution. We found that transcriptionally divergent dsRNA-responsive genes have higher rates of gene gain and loss (Fig. 4b) and consequently are also evolutionarily younger (Fig. 4c, Supplementary Fig. 17).

Previous reports have suggested that proteins encoded by younger genes tend to have fewer protein-protein interactions (PPIs) within cells ${ }^{42}$. Indeed, we found that rapidly diverging genes tend to have fewer PPIs (Fig. 4d). Together, these results suggest that transcriptionally divergent dsRNA-responsive genes evolve rapidly through various mechanisms, including fast coding sequence evolution and higher rates of gene loss and duplication events, and that their products have fewer interactions with other cellular proteins than those of less divergent genes.

The interaction between pathogens and the host immune system is thought to be an important driving force in the evolution of both sides. We therefore investigated the relationship between transcriptional divergence and interactions with viral proteins by compiling a data set of known host-virus interactions in humans ${ }^{6,43,44}$. Notably, genes whose products had no known viral interactions showed higher response divergence than genes encoding proteins with viral interactions (Fig. 4e). Furthermore, the transcriptional divergence of genes targeted by viral immunomodulators ${ }^{45}$ - viral proteins that subvert the host immune system-was lower still (Fig. 4e). These observations suggest that viruses have evolved to modulate the immune system by interacting with immune proteins that are relatively conserved in their response. Presumably, these genes cannot evolve away from viral interactions, unlike host genes that are less constrained ${ }^{46}$.

The summary of our results in Fig. 4 f highlights the differences in both regulatory and evolutionary characteristics between cytokines and other representative dsRNA-responsive genes. Cytokines evolve rapidly through various evolutionary mechanisms and have higher transcriptional variability across cells. By contrast, genes that are involved in immune response regulation, such as transcription factors and kinases, are more conserved and less heterogeneous across cells. These genes encode proteins that have more interactions with other cellular proteins, suggesting that higher constraints are imposed on their evolution. This group of conserved genes is more often targeted by viruses, revealing a relationship between host-pathogen dynamics and the evolutionary landscape of the innate immune response.

\section{Discussion}

Here, we have charted the evolutionary architecture of the innate immune response. We show that genes that diverge rapidly between 


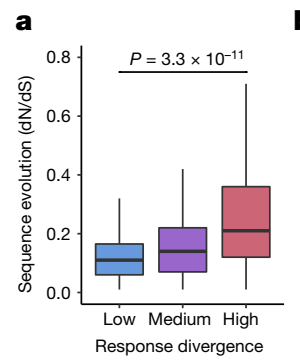

b

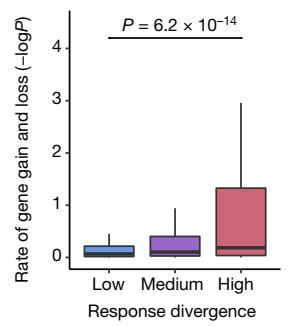

c

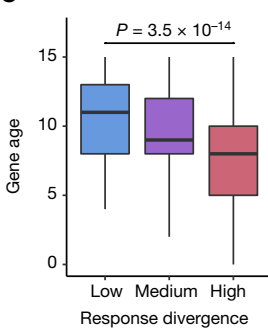

d

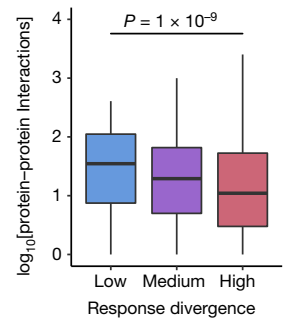

e

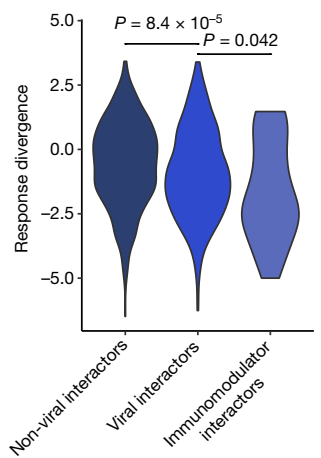

f
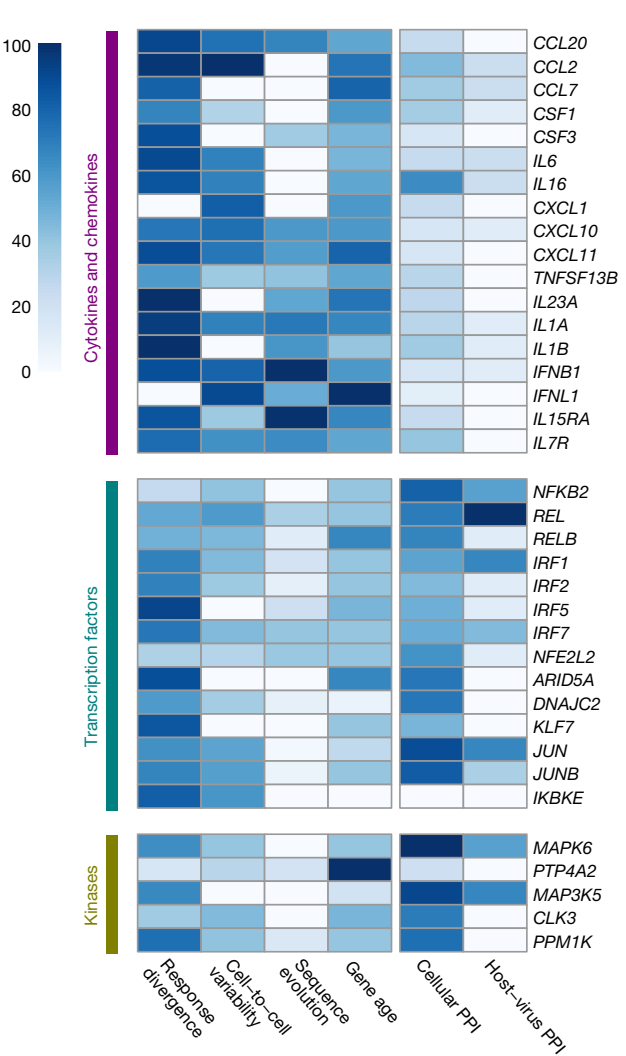

Fig. 4 | Relationship of response divergence and other evolutionary modes. a-d, dsRNA-responsive genes in fibroblasts are divided by level of response divergence into three groups, as in Fig. 1c. a, Coding sequence divergence, as measured using $\mathrm{dN} / \mathrm{dS}$ values across 29 mammals. Higher $\mathrm{dN} / \mathrm{dS}$ values denote faster coding sequence evolution ( $n=567$ genes). b, Rate at which genes were gained and lost within the gene family across the vertebrate clade (plotted as $-\log P$ ). Higher values denote faster gene gain and loss rate ( $n=955$ genes). c, Evolutionary age (estimated with Panther7 phylogeny and Wagner reconstruction algorithm). Values denote the branch number with respect to human (distance from human in the phylogenetic tree); higher values indicate greater age ( $n=931$ genes). d, Number of known physical interactions with other cellular proteins ( $n=955$ genes). e, Distribution of transcriptional response divergence values among dsRNA-responsive genes whose protein products do not interact with viral proteins, interact with at least one viral protein, or interact with viral immunomodulators $(n=648,307$ and 25 genes, respectively). a-e, One-sided Mann-Whitney tests. f, A scaled heat map showing values of response divergence (as in Fig. 1c), cell-to-cell variability (as in Fig. 3a), coding sequence divergence (dN/dS values, as in a), gene age (as in c; younger genes have darker colours), number of cellular PPIs (as in d) and number of host-virus interactions (as in e), for example genes from three functional groups: cytokines, transcription factors, and kinases. Values are shown in a normalized scale between 0 and 100, with the gene with the highest value assigned a score of 100. Missing values are shown in white. Boxplots represent the median, first quartile and third quartile with lines extending to the furthest value within $1.5 \times$ IQR. Violin plots show the kernel probability density of the data.

systems that have been exposed to continuous changes in external stimuli during evolution.

We have also shown that genes involved in regulation of the immune response-such as transcription factors and kinases-are relatively conserved in their transcriptional responses. These genes might be under stronger functional and regulatory constraints, owing to their roles in multiple contexts and pathways, which would limit their ability to evolve. This limitation could represent an Achilles' heel that is used by pathogens to subvert the immune system. Indeed, we found that viruses interact preferentially with conserved proteins of the innate immune response. Cytokines, on the other hand, diverge rapidly between species, owing to their promoter architecture and because they have fewer constraints imposed by intracellular interactions or additional variability in expression ${ }^{30,47}$. This finding suggests intriguing analogies between the mammalian immune and yeast stress responses-two 
non-immune functions. We therefore suggest that cytokines represent a successful host strategy to counteract rapidly evolving pathogens as part of the host-pathogen evolutionary arms race.

Cytokines also display high cell-to-cell variability and tend to be co-expressed with other cytokines and cytokine regulators in a small subset of cells, and this pattern is conserved across species. As prolonged or increased cytokine expression can result in tissue damage ${ }^{48-50}$, restriction of cytokine production to only a few cells may enable a rapid, but controlled, response across the tissue to avoid long-lasting and potentially damaging effects.

\section{Online content}

Any methods, additional references, Nature Research reporting summaries, source data, statements of data availability and associated accession codes are available at https://doi.org/10.1038/s41586-018-0657-2.

Received: 24 August 2017; Accepted: 17 August 2018; Published online 24 October 2018.

1. Borden, E. C. et al. Interferons at age 50: past, current and future impact on biomedicine. Nat. Rev. Drug Discov. 6, 975-990 (2007).

2. Iwasaki, A. A virological view of innate immune recognition. Annu. Rev. Microbiol. 66, 177-196 (2012)

3. Nielsen, R. et al. A scan for positively selected genes in the genomes of humans and chimpanzees. PLoS Biol. 3, e170 (2005).

4. Haygood, R., Babbitt, C. C., Fedrigo, O. \& Wray, G. A. Contrasts between adaptive coding and noncoding changes during human evolution. Proc. Natl Acad. Sci. USA 107, 7853-7857 (2010).

5. Fumagalli, M. et al. Signatures of environmental genetic adaptation pinpoint pathogens as the main selective pressure through human evolution. PLoS Genet. 7, e1002355 (2011).

6. Enard, D., Cai, L., Gwennap, C. \& Petrov, D. A. Viruses are a dominant driver of protein adaptation in mammals. eLife $\mathbf{5}$, e12469 (2016).

7. Barreiro, L. B. \& Quintana-Murci, L. From evolutionary genetics to human immunology: how selection shapes host defence genes. Nat. Rev. Genet. 11, $17-30(2010)$

8. Zhao, M., Zhang, J., Phatnani, H., Scheu, S. \& Maniatis, T. Stochastic expression of the interferon- $\beta$ gene. PLoS Biol. 10, e1001249 (2012).

9. Avraham, R. et al. Pathogen cell-to-cell variability drives heterogeneity in host immune responses. Cell 162, 1309-1321 (2015)

10. Shalek, A. K. et al. Single-cell RNA-seq reveals dynamic paracrine control of cellular variation. Nature 510, 363-369 (2014).

11. Hwang, S. Y. et al. Biphasic RLR-IFN- $\beta$ response controls the balance between antiviral immunity and cell damage. J. Immunol. 190, 1192-1200 (2013).

12. Porritt, R. A. \& Hertzog, P. J. Dynamic control of type I IFN signalling by an integrated network of negative regulators. Trends Immunol. 36, 150-160 (2015).

13. Ivashkiv, L. B. \& Donlin, L. T. Regulation of type I interferon responses. Nat. Rev. Immunol. 14, 36-49 (2014).

14. Brinkworth, J. F. \& Barreiro, L. B. The contribution of natural selection to present-day susceptibility to chronic inflammatory and autoimmune disease. Curr. Opin. Immunol. 31, 66-78 (2014).

15. Kobayashi, K. S. \& Flavell, R. A. Shielding the double-edged sword: negative regulation of the innate immune system. J. Leukoc. Biol. 75, 428-433 (2004)

16. Kumar, H., Kawai, T. \& Akira, S. Pathogen recognition by the innate immune system. Int. Rev. Immunol. 30, 16-34 (2011).

17. Barreiro, L. B., Marioni, J. C., Blekhman, R., Stephens, M. \& Gilad, Y. Functional comparison of innate immune signaling pathways in primates. PLOS Genet. $\mathbf{6}$, e1001249 (2010)

18. Schroder, K. et al. Conservation and divergence in Toll-like receptor 4-regulated gene expression in primary human versus mouse macrophages. Proc. Natl Acad. Sci. USA 109, E944-E953 (2012).

19. Shay, T. et al. Conservation and divergence in the transcriptional programs of the human and mouse immune systems. Proc. Natl Acad. Sci. USA 110, 2946-2951 (2013).

20. Brawand, D. et al. The evolution of gene expression levels in mammalian organs. Nature 478, 343-348 (2011)

21. Kalinka, A. T. et al. Gene expression divergence recapitulates the developmental hourglass model. Nature 468, 811-814 (2010).

22. Khaitovich, P., Enard, W., Lachmann, M. \& Pääbo, S. Evolution of primate gene expression. Nat. Rev. Genet. 7, 693-702 (2006).

23. Levin, M. et al. The mid-developmental transition and the evolution of animal body plans. Nature 531,637-641 (2016)

24. Reilly, S. K. \& Noonan, J. P. Evolution of gene regulation in humans. Annu. Rev Genomics Hum. Genet. 17, 45-67 (2016)

25. Tirosh, I., Weinberger, A., Carmi, M. \& Barkai, N. A genetic signature of interspecies variations in gene expression. Nat. Genet. 38, 830-834 (2006).

26. Haberle, V. \& Lenhard, B. Promoter architectures and developmental gene regulation. Semin. Cell Dev. Biol. 57, 11-23 (2016).

27. Lenhard, B., Sandelin, A. \& Carninci, P. Metazoan promoters: emerging characteristics and insights into transcriptional regulation. Nat. Rev. Genet. 13, 233-245 (2012).
28. Franz, K. M. \& Kagan, J. C. Innate immune receptors as competitive determinants of cell fate. Mol. Cell 66, 750-760 (2017).

29. Satija, R. \& Shalek, A. K. Heterogeneity in immune responses: from populations to single cells. Trends Immunol. 35, 219-229 (2014).

30. Newman, J. R. et al. Single-cell proteomic analysis of $S$. cerevisiae reveals the architecture of biological noise. Nature 441, 840-846 (2006).

31. Faure, A. J., Schmiedel, J. M. \& Lehner, B. Systematic analysis of the determinants of gene expression noise in embryonic stem cells. Cell Syst. 5, 471-484.e474 (2017).

32. Rand, $U$. et al. Multi-layered stochasticity and paracrine signal propagation shape the type-l interferon response. Mol. Syst. Biol. 8, 584 (2012).

33. Fumagalli, M. \& Sironi, M. Human genome variability, natural selection and infectious diseases. Curr. Opin. Immunol. 30, 9-16 (2014).

34. Johnson, W. E. \& Sawyer, S. L. Molecular evolution of the antiretroviral TRIM5 gene. Immunogenetics 61, 163-176 (2009).

35. Choo, S. W. et al. Pangolin genomes and the evolution of mammalian scales and immunity. Genome Res. 26, 1312-1322 (2016)

36. Braun, B. A., Marcovitz, A., Camp, J. G., Jia, R. \& Bejerano, G. Mx1 and Mx2 key antiviral proteins are surprisingly lost in toothed whales. Proc. Natl Acad. Sci. USA 112, 8036-8040 (2015).

37. $\mathrm{Xu}$, L. et al. Loss of RIG-I leads to a functional replacement with MDA5 in the Chinese tree shrew. Proc. Natl Acad. Sci. USA 113, 10950-10955 (2016).

38. Sackton, T. B., Lazzaro, B. P. \& Clark, A. G. Rapid expansion of immune-related gene families in the house fly, Musca domestica. Mol. Biol. Evol. 34, 857-872 (2017).

39. Brunette, R. L. et al. Extensive evolutionary and functional diversity among mammalian AIM2-like receptors. J. Exp. Med. 209, 1969-1983 (2012).

40. Malfavon-Borja, R., Wu, L. I., Emerman, M. \& Malik, H. S. Birth, decay, and reconstruction of an ancient TRIMCyp gene fusion in primate genomes. Proc. Natl Acad. Sci. USA 110, E583-E592 (2013).

41. Barber, M. F., Lee, E. M., Griffin, H. \& Elde, N. C. Rapid evolution of primate type 2 immune response factors linked to asthma susceptibility. Genome Biol. Evol. $\mathbf{9}$, 1757-1765 (2017).

42. Saeed, R. \& Deane, C. M. Protein-protein interactions, evolutionary rate, abundance and age. BMC Bioinformatics 7, 128 (2006).

43. Calderone, A., Licata, L. \& Cesareni, G. VirusMentha: a new resource for virus-host protein interactions. Nucleic Acids Res. 43, D588-D592 (2015).

44. Halehalli, R. R. \& Nagarajaram, H. A. Molecular principles of human virus protein-protein interactions. Bioinformatics 31, 1025-1033 (2015).

45. Pichlmair, A. et al. Viral immune modulators perturb the human molecular network by common and unique strategies. Nature 487, 486-490 (2012).

46. Dyer, M. D., Murali, T. M. \& Sobral, B. W. The landscape of human proteins interacting with viruses and other pathogens. PLoS Pathog. 4, e32 (2008).

47. Tirosh, I. \& Barkai, N. Two strategies for gene regulation by promoter nucleosomes. Genome Res. 18, 1084-1091 (2008).

48. Crow, Y. J. \& Manel, N. Aicardi-Goutières syndrome and the type interferonopathies. Nat. Rev. Immunol. 15, 429-440 (2015).

49. Hall, J. C. \& Rosen, A. Type I interferons: crucial participants in disease amplification in autoimmunity. Nat. Rev. Rheumatol. 6, 40-49 (2010).

50. Tisoncik, J. R. et al. Into the eye of the cytokine storm. Microbiol. Mol. Biol. Rev. 76, 16-32 (2012).

Acknowledgements We thank N. Eling, M. Fumagalli, Y. Gilad, O. Laufman, A. Marcovitz, J. Marioni, K. Meyer, M. Muffato, D. Odom, O. Stegle, A. Stern, M. Stubbington, V. Svensson and M. Ward for discussions; G. Emerton, A. Jinat, L. Mamanova, K. Polanski, A. Fullgrabe, N. George, S. Barnett, R. Boyd, S. Patel and C. Gomez for technical assistance; the Hipsci consortium for human fibroblast lines; and members of the Teichmann laboratory for support at various stages. This project was supported by ERC grants (ThDEFINE, ThSWITCH) and an EU FET-OPEN grant (MRG-GRAMMAR No 664918) and Wellcome Sanger core funding (Grant No WT206194). T.H. was supported by an HFSP Long-Term Fellowship and by EMBO Long-Term and Advanced fellowships. V.P. is funded by Fondazione Umberto Veronesi.

Reviewer information Nature thanks L. Barreiro, I. Yanai and the other anonymous reviewer(s) for their contribution to the peer review of this work.

Author contributions T.H. and S.A.T. designed the project; T.H., X.C., R.J.M., R.R., N.K. and J.-E.P. performed experiments with help from V.P., G.D. and F.A.V.B.; T.H., X.C., R.J.M., R.R., T.G. and J.H. analysed the data with help from G.N., L.B.-C., G.T, A.N. and M.L.; J.F., E.S., P.V. I.K., M.D. and M.H. provided samples; S.A.T. supervised the project; T.H., R.R., N.K. and S.A.T. wrote the manuscript with input from all authors.

Competing interests The authors declare no competing interests.

\section{Additional information}

Extended data is available for this paper at https://doi.org/10.1038/s41586018-0657-2.

Supplementary information is available for this paper at https://doi.org/ 10.1038/s41586-018-0657-2.

Reprints and permissions information is available at http://www.nature.com/ reprints.

Correspondence and requests for materials should be addressed to T.H. or S.A.T.

Publisher's note: Springer Nature remains neutral with regard to jurisdictional claims in published maps and institutional affiliations. 


\section{METHODS}

Ethical compliance. This project was approved by the Wellcome Sanger Institute Animal Welfare and Ethical Review Body, and complied with all relevant ethical regulations regarding animal research and human studies. Human cells were obtained from the Hipsci project ${ }^{51}$, where they were collected from volunteers recruited from the NIHR Cambridge BioResource (written consent was given). Human skin profiling was performed in accordance with protocols approved by the Newcastle Research Ethics Committee (REC approval 08/H0906/95+5). Macaque skin samples were obtained from animals assigned to unrelated noninfectious studies, provided by Public Health England's National Infection Service in accordance with Home Office (UK) guidelines and approved by the Public Health England Ethical Review Committee under an appropriate UK Home Office project license.

Cross-species dermal fibroblast stimulation with dsRNA and IFNB. Tissue culture. We cultured primary dermal fibroblasts from low passage cells (below 10) that originated from females from four different species (human (European ancestry), rhesus macaque, C57BL/6 (black 6) mouse and brown Norway rat). All skin samples were taken from shoulders. Stimulation experiments and library preparations were done in identical conditions across all species and for all genomics techniques. Details on the numbers of individuals used in each technique are listed in each technique's section and in Supplementary Table 1.

Human cells were obtained from the Hipsci project ${ }^{51}$ (http://www.hipsci.org/). Rhesus macaque cells were extracted from skin tissues that were incubated for $2 \mathrm{~h}$ with $0.5 \%$ collagenase B (Roche; 11088815001) after mechanical processing, and then filtered through $100-\mu \mathrm{m}$ strainers before being plated and passaged before cryo-banking. Rodent cells were obtained from PeloBiotech where they were extracted using a similar protocol. In vitro cultured fibroblasts from all four species resemble a particular in vivo cluster of dermal fibroblasts (see Supplementary Information). Cells were not tested for mycoplasma contamination.

Prior to stimulation, cells were thawed and grown for several days in ATCC fibroblast growth medium (Fibroblast Basal Medium (ATCC, ATCC-PCS-201-030) with Fibroblast Growth Kit-Low serum (ATCC, PCS-201-041) (supplemented with Primocin (Invivogen, ant-pm-1) and penicillin/streptomycin (Life Technologies, 15140122)) - a controlled medium that has proven to provide good growing conditions for fibroblasts from all species, with slightly less than $24 \mathrm{~h}$ doubling times. About $18 \mathrm{~h}$ before stimulation, cells were trypsinized, counted and seeded into 6 -well plates (100,000 cells per well). Cells were stimulated as follows: (1) stimulated with $1 \mu \mathrm{g} / \mathrm{ml}$ high-molecular mass poly(I:C) (Invivogen,tlrl-pic) transfected with $2 \mu \mathrm{g} / \mathrm{ml}$ Lipofectamin 2,000 (ThermoFisher, 11668027); (2) mock transfected with Lipofectamin 2,000; (3) stimulated with 1,000 IU of IFNB for $8 \mathrm{~h}$ (human IFNB: 11410-2 (for human and macaque cells); rat IFNB: 13400-1; mouse IFNB: 12401-1; all IFNs were obtained from PBL, and had activity units based on similar virological assays); or (4) left untreated. Interferon stimulation was used as a control, to study how genes that were upregulated in the secondary wave of the innate immune response diverge between species.

Additional human and mouse samples were stimulated with 1,000 IU of cross-mammalian IFN (CMI, or Universal Type I IFN Alpha, PBL, 11200-1). The latter stimulation was done to assess the effects of species-specific and batchspecific IFNB.

In all of the above-mentioned stimulations, we used a longer time course for single-cell RNA-seq than for bulk RNA-seq, for two main reasons: (1) in the bulk, we chose to focus on one main stimulation time point for simplicity and to obtain an intuitive fold-change between stimulated and unstimulated conditions; (2) in single cells, when studying cell-to-cell variability, we chose to profile, in addition to the main stimulation time point, cells in earlier and later stages of the response. This is important for studying how the dynamics and magnitude of the response affect gene expression variability between responding cells.

The poly(I:C) we used tested negative for the presence of bacterial beta-endotoxin using a coagulation test (PYROGENT Plus, $0.06 \mathrm{EU} / \mathrm{ml}$ sensitivity, N283-06). Bulk RNA-seq: library preparation and sequencing. For bulk transcriptomics analysis, cells from individuals from different species were grown in parallel and stimulated with dsRNA, IFNB (and cross-mammalian IFN) and their respective controls. In total, samples from 6 humans, 6 macaques, 3 mice and 3 rats were used. Total RNA was extracted using the RNeasy Plus Mini kit (Qiagen, 74136), using QIAcube (Qiagen). RNA was then measured using a Bioanalyzer 2100 (Agilent Technologies), and samples with RIN $<9$ were excluded from further analysis (one macaque sample stimulated with poly(I:C) and its control).

Libraries were produced using the Kapa Stranded mRNA-seq Kit (Kapa Biosystems, KK8421). The Kapa library construction protocol was modified for automated library preparation by Bravo (Agilent Technologies). cDNA was amplified in 13 PCR cycles, and purified using Ampure XP beads (Beckman Coulter, A63882) (1.8 $\times$ volume) using Zephyr (Perkin Elmer). Pooled samples were sequenced on an Illumina HiSeq 2500 instrument, using paired-end 125 -bp reads.
ChIP-seq: library preparation and sequencing. Samples from three individuals from each of the four species were grown and stimulated (with poly(I:C) for $4 \mathrm{~h}$ or left untreated, as described above) in parallel to samples collected for bulk RNA-seq. Following stimulation, samples were crosslinked in $1 \% \mathrm{HCHO}$ (prepared in $1 \times$ DPBS) at room temperature for $10 \mathrm{~min}$, and $\mathrm{HCHO}$ was quenched by the addition of glycine at a final concentration of $0.125 \mathrm{M}$. Cells were pelleted at $4^{\circ} \mathrm{C}$ at 2,000g, washed with ice-cold $1 \times$ DPBS twice, and snap-frozen in liquid nitrogen. Cell pellets were stored at $-80^{\circ} \mathrm{C}$ until further stages were performed. ChIPmentation was performed according to version 1.0 of the published proto$\mathrm{col}^{52}$ with a few modifications (see additional details in Supplementary Methods).

Library preparation reactions contained the following reagents: $10 \mu \mathrm{l}$ purified DNA (from the above procedure), $2.5 \mu \mathrm{l}$ PCR Primer Cocktails (Nextera kit, Illumina, FC-121-1030), $2.5 \mu \mathrm{l}$ N5xx (Nextera index kit, Illumina FC-121-1012), $2.5 \mu 1$ N7xx (Nextera index kit, Illumina, FC-121-1012), $7.5 \mu 1$ NPM PCR Master Mix (Nextera kit, Illumina, FC-121-1030). PCR cycles were as follows: $72^{\circ} \mathrm{C}, 5 \mathrm{~min}$; $98^{\circ} \mathrm{C}, 2 \mathrm{~min} ;\left[98^{\circ} \mathrm{C}, 10 \mathrm{~s}, 63^{\circ} \mathrm{C}, 30 \mathrm{~s}, 72^{\circ} \mathrm{C}, 20 \mathrm{~s}\right] \times 12 ; 10^{\circ} \mathrm{C}$ hold.

Amplified libraries were purified by double AmpureXP bead purification: first with $0.5 \times$ bead ratio, keep supernatant, second with $1.4 \times$ bead ratio, keep bound DNA. Elution was done in $20 \mu \mathrm{l}$ Buffer EB (QIAGEN).

One microlitre of library was run on a Bioanalyzer (Agilent Technologies) to verify normal size distribution. Pooled samples were sequenced on an Illumina HiSeq 2000 instrument, using paired-end 75-bp reads.

Flow cytometry for single-cell RNA-seq. For scRNA-seq, we performed two biological replicates, with each replicate having one individual from each of the four studied species. A time course of dsRNA stimulation of 0,4 , and $8 \mathrm{~h}$ was used in one replicate (divided into two technical replicates), while the second replicate included a time course of $0,2,4$, and $8 \mathrm{~h}$. Poly(I:C) transfection was done as described above. In the case of sorting with IFNLUX, we used rhodamine-labelled poly(I:C).

Cells were sorted with either Beckman Coulter MoFlo XDP (first replicate) or Becton Dickinson INFLUX (second replicate) into wells containing $2 \mu 1$ lysis buffer (1:20 solution of RNase Inhibitor (Clontech, 2313A) in $0.2 \% \mathrm{v} / \mathrm{v}$ Triton $\mathrm{X}-100$ (Sigma-Aldrich, T9284)), spun down and immediately frozen at $-80^{\circ} \mathrm{C}$.

When sorting with MoFlo, a pressure of 15 psi was used with a $150-\mu \mathrm{m}$ nozzle, using the 'Single' sort purity mode. Dead or late-apoptosis cells were excluded using propidium iodide at $1 \mu \mathrm{g} / \mathrm{ml}$ (Sigma, Cat Number P4170) and single cells were selected using FSC W versus FSC $\mathrm{H}$. When sorting with INFLUX, a pressure of 3 psi was used with a $200-\mu \mathrm{m}$ nozzle, with the 'single' sort mode. Dead or late-apoptosis cells were excluded using $100 \mathrm{ng} / \mathrm{ml}$ DAPI (4',6-diamidino-2phenylindole) (Sigma, D9542). DAPI was detected using the 355-nm laser (50 $\mathrm{mW}$ ), using a $460 / 50 \mathrm{~nm}$ bandpass filter. Rhodamine was detected using the $561-\mathrm{nm}$ laser $(50 \mathrm{~mW})$, using a $585 / 29 \mathrm{~nm}$ bandpass filter. Single cells were collected using FSC W versus FSC $\mathrm{H}$.

Library preparation from full-length RNA from single cells and sequencing. Sorted plates were processed according to the Smart-seq 2 protocol ${ }^{53}$. Oligo-dT primer (IDT), dNTPs (ThermoFisher, 10319879) and ERCC RNA Spike-In Mix (1:25,000,000 final dilution, Ambion, 4456740) were added to each well, and reverse transcription (using $50 \mathrm{U}$ SmartScribe, Clontech, 639538) and PCR were performed following the original protocol with 25 PCR cycles. cDNA libraries were prepared using Nextera XT DNA Sample Preparation Kit (Illumina, FC-131-1096), according to the protocol supplied by Fluidigm (PN 100-5950 B1). Quality Checks on cDNA were done using a Bioanalyser 2100 (Agilent Technologies). Libraries were quantified using the LightCycler 480 (Roche), pooled and purified using AMPure XP beads (Beckman Coulter) with Hamilton 384 head robot (Hamilton Robotics). Pooled samples were sequenced on an Illumina HiSeq 2500 instrument, using paired-end 125 -bp reads.

Read mapping to annotated transcriptome. For bulk RNA-seq samples, adaptor sequences and low-quality score bases were first trimmed using Trim Galore (version 0.4.1) (with the parameters '-paired-quality 20-length 20 -e 0.1 -adapter AGATCGGAAGAGC'). Trimmed reads were mapped and gene expression was quantified using Salmon (version 0.6.0) ${ }^{54}$ with the following command: 'salmon quant -i [index_file_directory] -1 ISR -p 8-biasCorrect-sensitive-extraSensitive -o [output_directory] -1 -g [ENSEMBL_transcript_to_gene_file]-useFSPDnumBootstraps 100'. Each sample was mapped to its respective species' annotated transcriptome (downloaded from ENSEMBL, version 84: GRCh38 for human, MMUL_1 for macaque, GRCm38 for mouse, Rnor_6.0 for rat). We included only the set of coding genes (*.cdna.all.fa files). We removed annotated secondary haplotypes of human genes by removing genes with 'CHR_HSCHR'.

Quantifying differential gene expression in response to dsRNA. To quantify differential gene expression between treatment and control for each species and for each treatment separately, we used edgeR (version 3.12.1) ${ }^{55}$ using the rounded estimated counts from Salmon. This was done only for genes that had a significant level of expression in at least one of the four species (TPM $>3$ in at least $N-1$ libraries, where $N$ is the number of different individuals we have for this species with libraries that passed quality control, and TPM is transcripts per million). Differential 
expression analysis was performed using the edgeR exact test, and $P$ values were adjusted for multiple testing by estimating the false discovery rate (FDR). Conservation and divergence in immune response: fold-change-based analysis. We compared the overall change in response to treatment (dsRNA or IFNB) between pairs of species, by computing the Spearman correlation of the fold-change in response to treatment across all one-to-one orthologues that were expressed in at least one species (Extended Data Fig. 1a-h). Fold-change was calculated with edgeR, as described above. Spearman correlations of all expressed genes appear in grey. Correlations of the subset of differentially expressed genes (genes with FDR-corrected $P<0.01$ in at least one of the compared species) appear in black.

In Extended Data Fig. 1a-c, we show comparisons in response to dsRNA. In Extended Data Fig. 1d-f, we show comparisons in response to IFNB, which we use here to study the similarity of the secondary immune response between species.

We constructed a tree based on a gene's change in expression in response to dsRNA and to IFNB, using expressed genes that had one-to-one orthologues across all four species and were expressed in at least one species in at least one condition (Extended Data Fig. 1i). We used hierarchical clustering, with the hclust command from the stats $\mathrm{R}$ package, with the distance between samples computed as $1-\rho$, where $\rho$ is the pairwise Spearman correlation between each pair of species mentioned above (a greater similarity, reflected in a higher correlation, results in a smaller distance) and 'average' as the clustering method.

The above-mentioned analyses focus on one-to-one orthologues between the compared species. In Supplementary Table 6, we quantify the similarity in response between species (based on Spearman correlations) when adding genes with one-to-many orthologues.

Quantifying gene expression divergence in response to immune challenge. To quantify transcriptional divergence in immune response between species, we focus on genes that have annotated one-to-one orthologues across the studied species (human, macaque, mouse and rat). 9,753 of the expressed genes have annotated one-to-one orthologues in all four species, out of which 955 genes are differentially expressed in human in response to dsRNA treatment (genes with an FDR-corrected $P<0.01$ ).

We define a measure of response divergence (based on a previous study ${ }^{56}$ ) by calculating the differences between the fold-change estimates across the orthologues: response divergence $=\log \left[1 / 4 \times \sum_{i, j}\left(\log \left[\text { FC primate }_{i}\right]-\log [\text { FC rodent }]_{j}\right)^{2}\right]$. This measure takes into account the structure of the phylogeny, and gives a relative measure of divergence in response across all genes with one-to-one orthologues.

To consider differences between species, we focus on between-clade differences (primates versus rodents), rather than on within-clade differences. In this way, we map the most significant macro-evolutionary differences along the longest branches of our four-species phylogeny. In addition, averaging within clades acts as a reduction of noise ${ }^{56}$

We compared this divergence measure to two other measures that use models (and incorporate both between- and within-clade divergence) and found a strong correlation between the divergence estimates across the three approaches (Supplementary Figs. 3, 4).

In most of the subsequent analyses, we focus on the 955 dsRNA-responsive genes: genes that were differentially expressed in response to dsRNA (genes that have an FDR-corrected $P<0.01$ in human, and have annotated one-to-one orthologues in the other three species). For some of the analyses, we split these 955 genes based on quartiles, into genes with high, medium and low divergence (Fig. 1c).

We also studied how imprecisions in the fold-change estimates affected the response divergence estimates and subsequent analyses (Supplementary Figs. 5, 6). Comparison of response divergence between different functional groups. To compare the divergence rates between sets of dsRNA-responsive genes that have different functions in the innate immune response, we split these 955 genes into the following functional groups (all groups are mutually exclusive, and any gene that belongs to two groups was excluded from the latter group; human gene annotations were used).

We first grouped genes by annotated molecular functions: viral sensors (genes that belong to one of the GO categories: GO:0003725 (dsRNA binding), GO:0009597 (detection of virus), and GO:0038187 (pattern recognition receptor activity)); cytokines, chemokines and their receptors (GO:0005125 (cytokine activity), GO:0008009 (chemokine activity), GO:0004896 (cytokine receptor activity), and GO:0004950 (chemokine receptor activity)); transcription factors (taken from the Animal Transcription Factor DataBase (version 2.0 $)^{57}$ ); chromatin modulators (GO:0016568 (chromatin modification), GO:0006338 (chromatin remodelling), GO:0003682 (chromatin binding), and GO:0042393 (histone binding)); kinases and phosphatases (GO:0004672 (protein kinase activity) and GO:0004721 (phosphoprotein phosphatase activity)); ligases and deubiquitinases (GO:0016579 (protein deubiquitination), GO:0004842 (ubiquitin-protein transferase activity) and GO:0016874 (ligase activity)); and other enzymes (mostly involved in metabolism rather than regulation: GO:0003824 (catalytic activity)). The divergence response values of these functional subsets were compared to the entire group of 955 dsRNA-responsive genes (Fig. 2d, e).
Next, we grouped genes by biological processes that are known to be important in the innate immune response: antiviral defence (GO:0051607 (defence response to virus)); inflammation (GO:0006954 (inflammatory response)); apoptosis (GO:0006915 (apoptotic process)); and regulation (GO annotations related to regulation of innate immune response pathways include only few genes. We thus used as the group of genes related to regulation, the merged group of genes that are annotated as transcription factors, chromatin modulators, kinases and phosphates or ligases and deubiquitinases, since all these groups include many genes that are known to regulate the innate immune response.)

Gene lists belonging to the mentioned GO annotations were downloaded using QuickGo ${ }^{58}$. The distribution of response divergence values for each of the functional groups was compared with the distribution of response divergence of the entire set of dsRNA-responsive genes. Cytokines, chemokines and their receptors are merged in Fig. 2d, e, 3c. Analogous comparisons of functional groups in IFNB response (with 841 IFNB-responsive genes) are shown in Supplementary Fig. 1. See additional analyses in Supplementary Information.

Alignment and peak calling of ChIP-seq reads. ChIP-seq reads were trimmed using trim_galore (version 0.4.1) with '-paired-trim1-nextera' flags. The trimmed reads were aligned to the corresponding reference genome (hg38 for human, rheMac2 for macaque, $\mathrm{mm} 10$ for mouse, $\mathrm{rn} 6$ for rat; all these genomes correspond to the transcriptomes used for RNA-seq mapping) from the UCSC Genome Browser ${ }^{59}$ using bowtie2 (version 2.2.3) with default settings ${ }^{60}$. In all four species, we removed the $\mathrm{Y}$ chromosome. In the case of human, we also removed all alternative haplotype chromosomes. Following alignment, low-confident mapped and improperly paired reads were removed by samtools ${ }^{61}$ with ' $-\mathrm{q} 30$ - $\mathrm{f} 2$ ' flags.

Enriched regions (peaks) were called using MACS2 (v.2.1.1) ${ }^{62}$ with a corrected $P$ value cutoff of 0.01 with '- $f$ BAMPE -q 0.01 -B-SPMR' flags, using input DNA as control. The genome sizes (the argument for '-g' flag) used were 'hs' for human, ' $\mathrm{mm}$ ' for mouse, $3.0 \times 10^{9}$ for macaque and $2.5 \times 10^{9}$ for rat. Peaks were considered reproducible when they were identified in at least two of the three biological replicates and overlapped by at least $50 \%$ of their length (non-reproducible peaks were excluded from subsequent analyses). Reproducible peaks were then merged to create consensus peaks from overlapping regions of peaks from the three replicates by using mergeBed from the bedtools suite ${ }^{63}$.

Gene assignment and conservation of active promoters and enhancers. We subsequently linked human peaks with the genes they might be regulating as follows: $\mathrm{H} 3 \mathrm{~K} 4 \mathrm{me} 3$ consensus peak was considered the promoter region of a given gene if its centre was between $2 \mathrm{~kb}$ upstream and $500 \mathrm{bp}$ downstream of the annotated TSS of the most abundantly expressed transcript of that gene.

Similarly, H3K27ac was considered the enhancer region of a given gene if its centre was in a distance above $1 \mathrm{~kb}$ and below $1 \mathrm{Mb}$, and there was no overlap (of 1 bp or more) with any H3K4me3 peak.

In each case where, based on the distance criteria, more than a single peak was linked to a gene (or more than a single gene was linked to a peak), we took only the closest peak-gene pair (ensuring that each peak will have up to one gene and vice versa).

To compare active promoters and enhancers between species, we excluded any human peak that could not be uniquely mapped to the respective region in the other species. This was done by looking for syntenic regions of human peaks in the other three species by using liftOver ${ }^{64}$, and removing peaks that had either unmapped regions or more than one mapped region in the compared species. We considered syntenic regions with at least $70 \%$ sequence similarity between the species (minMatch $=0.7$, and 0.8 in the case of human-macaque comparison), with a minimal length (minSizeQ and minSizeT) corresponding to the length of the shortest peak (128 bp in H3K4 and 142 bp in H3K27).

We defined an active human promoter or enhancer as conserved if a peak was identified in the corresponding region of the other species (we repeated this analysis by comparing human with each of the other three species separately). We compared the occurrence of conserved promoters and enhancers in genes that are highly divergent in response to dsRNA with low-divergence genes, and used Fisher's exact test to determine the statistical significance of the observed differences between high- and low-divergence genes (Extended Data Fig. 2).

Promoter sequence analysis. To calculate the total number of transcription factor binding motifs in a gene's active promoter region, we downloaded the nonredundant JASPAR core motif matrix (pfm_vertebrates.txt) from the JASPAR 2016 server $^{65}$ and searched for significant matches for these motifs using FIMO $^{66}$ in human $\mathrm{H} 3 \mathrm{~K} 4 \mathrm{me} 3$ peaks. The TFBM density of peaks was calculated by dividing the total number of motif matches in a peak by the peak's length. TBFM density values in human $\mathrm{H} 3 \mathrm{~K} 4 \mathrm{me} 3$ peaks linked with high- and low-divergence genes were compared (Fig. 2a).

PhyloP7 values were used to assess promoter sequence conservation ${ }^{67}$. Sequence conservation quantification was performed by taking the estimated nucleotide substitution rate for each nucleotide along the promoter sequence ( $500 \mathrm{bp}$ upstream of the TSS of the relevant human gene). When several annotated transcripts existed, 
the TSS of the most abundantly expressed transcript was used (based on bulk RNA data). The substitution rate values from all genes were aligned, based on their TSS position, and a mean for each of the 500 positions was calculated separately for the group of genes with high, medium and low response divergence. The two-sample Kolmogorov-Smirnov test was used to compare the paired distribution of rates between the means of the high-divergence and low-divergence sets of genes. To plot the mean values of the three sets of divergent genes, the geom_smooth function from the ggplot2 $\mathrm{R}$ package was used with default parameters (with loess as the smoothing method) (Fig. 2b).

Human CGI annotations were downloaded from the UCSC genome table browser (hg38), and CGI genes were defined as those with a CGI overlapping their core promoter (300 bp upstream of the TSS reference position, and $100 \mathrm{bp}$ downstream of it, as suggested previously ${ }^{18}$ ). Genes were defined as having a TATA box if they had a significant match to the Jaspar TATA box matrix (MA0108.1) in the $100 \mathrm{bp}$ upstream of their TSS by FIMO ${ }^{66}$ with default settings (we used a $100 \mathrm{bp}$ window owing to possible inaccuracies in TSS annotations). We note that only 28 out of 955 dsRNA-responsive genes had a matching TATA-box motif in this region. For both TATA and CGI analyses, the promoter sequences of the human orthologues were used.

Read mapping and quality control of scRNA-seq (full-length RNA). Gene expression was quantified in a manner similar to the quantification for bulk transcriptomics libraries described above. Low-quality cells were filtered using quality control criteria (cells with at least 100,000 mapped reads, with at least 2,000 expressed genes with TPM $>3$, with ERCC $<10 \%$ and MT $<40 \%$, where ERCC and MT refer to reads mapped to synthetic RNA Spike-In genes and mitochondrial genes). This quality control filtering resulted in 240 cells from a first biological replicate, including two technical replicates (with a time course of $0,4,8 \mathrm{~h}$ ). In a second larger biological replicate (with a dsRNA stimulation time course of $0,2,4,8 \mathrm{~h}$ ), 728 cells passed quality control. Results throughout the manuscript relate to the second cross-species biological replicate in which a higher proportion of cells passed QC, and the lower-quality first replicate data were not considered further.

Cell-to-cell variability analysis. To quantify the biological cell-to-cell variability of genes, we applied the DM (Distance to Median) approach-an established method, which calculates the cell-to-cell variability in gene expression while accounting for confounding factors such as gene expression level ${ }^{30}$. This is done by first filtering out genes that are expressed at low levels: for Smart-seq2 data we included only genes that had an average expression of at least 10 size-factor normalized reads (except for Extended Data Fig. 9a, in which we reduced the threshold to 5, to allow a larger number of genes to be included in the comparisons). This procedure was done to filter genes that displayed higher levels of technical variability between samples owing to low expression. Second, to account for gene expression level, the observed cell-to-cell variability of each gene was compared with its expected variability, based on its mean expression across all samples and in comparison with a group of genes with similar levels of mean expression. This $\mathrm{DM}$ value is also corrected by gene length (in the case of Smart-seq2 data), yielding a value of variability that can be compared across genes regardless of their length and mean expression values ${ }^{68}$. As a second approach, we used BASiCS ${ }^{69,70}$ (see Supplementary Information).

We note that the relationship observed in Fig. 3a between response divergence and cell-to-cell variability is not an artefact, stemming from differences in expression levels: (A) With respect to cell-to-cell variability, a gene's expression level is controlled for by DM calculations, where expression level is regressed by using a running median (Supplementary Fig. 14). (B) Similarly, we can regress the expression level measured in bulk RNA-seq from the quantified response divergence by subtracting the running median of expression from the divergence estimates. When repeating the analysis comparing cell-to-cell variability versus regressed response divergence, the relationship between the two is maintained (Supplementary Fig. 15).

Cytokine co-expression analysis. For the chemokine gene CXCL10, we built a network (using CytoScape ${ }^{71}$ ) of genes that correlate with CXCL10 in dsRNAstimulated human fibroblasts and in at least one more species, using genes with a Spearman correlation value above 0.3 (see Fig. 3d and Supplementary Information)

Coding sequence evolution analysis. The ratio $\mathrm{dN} / \mathrm{dS}$ (non-synonymous to synonymous codon substitutions) of human genes across the mammalian clade was obtained from a previous study that used orthologous genes from 29 mammals $^{72}$. Distributions of $\mathrm{dN} / \mathrm{dS}$ values were computed for each of the three groups of genes with low, medium and high divergence in response to dsRNA, and are plotted in Fig. 4a.

Rate of gene gain and loss analysis. The significance at which a gene's family has experienced a higher rate of gene gain and loss in the course of vertebrate evolution, in comparison with other gene families, was retrieved from ENSEMBL ${ }^{73}$. The statistics provided by ENSEMBL are calculated using the CAFE method ${ }^{74}$, which estimates the global birth and death rate of gene families and identifies gene families that have accelerated rates of gain and loss. Distributions of the $P$ values from this statistic were computed for each of the three groups of genes with low, medium and high divergence in response to dsRNA and are plotted as the negative logarithm values in Fig. 4b.

Gene age analysis. Gene age estimations were obtained from ProteinHistorian ${ }^{75}$. To ensure that the results were not biased by a particular method of ancestral protein family reconstruction or by specific gene family assignments, we used eleven different estimates for mammalian genes (combining five different databases of protein families with two different reconstruction algorithms for age estimation, as well as an estimate from the phylostratigraphic approach). For each gene, age was defined with respect to the species tree, where a gene's age corresponds to the branch in which its family is estimated to have appeared (thus, larger numbers indicate evolutionarily older genes).

Data for gene age in comparison with divergence in response to dsRNA are shown in Fig. 4c (using Panther7 phylogeny and Wagner reconstruction algorithm) and in Supplementary Fig. 17a (for all 11 combinations of gene family assignments and ancestral family reconstructions). See additional analyses in Supplementary Information.

Cellular protein-protein interaction analysis. Data on the number of experimentally validated PPIs for human genes were obtained from STRING (version 10) ${ }^{76}$ Distributions of PPIs for genes with low, medium and high divergence in response to dsRNA are plotted in Fig. $4 \mathrm{~d}$.

Host-virus interaction analysis. Data on host-virus protein-protein interactions were downloaded from the VirusMentha database ${ }^{43}$, and combined with two additional studies that have annotated host-virus protein-protein interactions ${ }^{6,44}$. We split the 955 dsRNA-responsive genes into genes with known viral interactions (genes whose protein products were reported to interact with at least one viral protein), and genes with no known viral interactions: 'viral interactors' and 'no viral interactions', respectively, in Fig. 4e. In addition, we define a subset of genes within the viral interactors set: those known to interact with viral proteins that are immunomodulators (proteins known to target the host immune system and modulate its response ${ }^{45}$ ).

We note that the results presented in Fig. 4e are in agreement with previous analyses that are based on all human genes and on coding sequence evolution ${ }^{46}$. However, the overlap in the sets of genes between the previous analyses and the one presented here is small (for example, in one published study ${ }^{46}$ there were 535 human genes with known interactions with pathogens, 57 of which overlap with the 955 genes that are the basis of the current analysis).

Additional experiments with human fibroblasts and human skin tissue. Additional experiments were performed with human dermal fibroblasts and with cells extracted from human skin tissues to study in greater detail the relationship between response divergence across species and cell-to-cell variability. See Supplementary Methods and Supplementary Discussion for details.

Cross-species bone marrow-derived phagocyte stimulation with LPS and dsRNA. Tissue culture. Primary bone marrow-derived mononuclear phagocytes originating from females of four different species (black 6 mouse, brown Norway rat, rabbit and pig) and cultured with GM-CSF, were obtained from PeloBiotech. Twenty-four hours before the start of the stimulation time course, cells were thawed and split into 12-well plates (500,000 cells per well). Cells were stimulated with: (1) $100 \mathrm{ng} / \mathrm{ml}$ LPS (Invivogen, tlrl-smlps), or with (2) $1 \mu \mathrm{g} / \mathrm{ml}$ high-molecular mass poly(I:C) (Invivogen, tlrl-pic) transfected with $2 \mu \mathrm{l} / \mathrm{ml}$ Lipofectamin 2,000 (ThermoFisher, 11668027). LPS stimulation time courses of $0,2,4,6 \mathrm{~h}$ were performed for all species. Poly(I:C) stimulations were performed for rodents for $0,2,4,6 \mathrm{~h}$. We also processed cells for bulk RNA-seq for 0 and $4 \mathrm{~h}$ stimulation time points. Details on the individuals used in each technique are listed in Supplementary Table 2.

Library preparation for single cells using microfluidic droplet cell capture. Following stimulation, cells were collected using Cell Dissociation Solution Non-enzymatic (Sigma-Aldrich, C5914), washed and resuspended in $1 \times$ PBS with $0.5 \%(\mathrm{w} / \mathrm{v})$ BSA. Cells were then counted and loaded on the 10x Chromium machine aiming for a targeted cell recovery of 5,000 cells according to the manual. Libraries were prepared following the Chromium Single Cell $3^{\prime}$ v2 Reagent Kit Manual ${ }^{77}$. Libraries were sequenced on an Illumina HiSeq 4000 instrument with $26 \mathrm{bp}$ for read 1 and $98 \mathrm{bp}$ for read 2.

Library preparation and sequencing for bulk RNA-seq. Total RNA was extracted and libraries were prepared as described in the fibroblasts section. Pooled samples were sequenced on an Illumina HiSeq 4000 instrument, using paired-end 75-bp reads. Quantifying gene expression in bulk RNA-seq data. Adaptor sequences and low-quality score bases were trimmed using Trim Galore (version 0.4.1). Trimmed reads were mapped and gene expression was quantified using Salmon: (version $0.9 .1)^{54}$ with the following command: 'salmon quant $-\mathrm{i}$ [index_file_directory] / -1 ISR -p 8-seqBias-gcBias-posBias -q -o [output_directory] - 1 -g [ENSEMBL_ transcript_to_gene_file]-useVBOpt-numBootstraps 100'. Mouse samples were mapped to mouse transcriptome (ENSEMBL, version 84). We note that we used 
the bulk data only for TSS analysis. For differential expression analysis, we used an in silico bulk from the single-cell data (see below).

Quantifying gene expression in microfluidic droplet cell capture data. Microfluidic droplet cell capture data was first quantified using 10x Genomics' Cell Ranger Single-Cell Software Suite (version 2.0, $10 \times$ Genomics Inc.) ${ }^{77}$ against the relevant genome (ENSEMBL, version 84). We removed cells with fewer than 200 genes or more than $10 \%$ mitochondrial reads. To remove potential doublets, we excluded the top $10 \%$ of cells expressing the highest numbers of genes. Genes expressed in less than $0.5 \%$ of the cells were excluded from the calculations. We then filtered cells that expressed fewer than $10 \%$ of the total number of filtered genes.

Since bone marrow-derived phagocytes may include secondary cell populations, we focused our analysis on the major cell population. We identified clusters within each data set, using the Seurat ${ }^{78}$ functions RunPCA, followed by FindClusters (using 20 dimensions from the PCA, default perplexity and a resolution of 0.1) and have taken the cells belonging to the largest cluster for further analysis, resulting in a less heterogeneous population of cells. A lower resolution of 0.03 was used for rabbit-LPS4, rabbit-LPS2, mouse-PIC2, mouse-PIC4; and 0.01 for rabbit-LPS6.

Quantifying gene expression divergence in response to immune challenge. We created an in silico bulk table by summing up the UMIs of the post-QC single cells belonging to the largest cluster of cells, in each of the samples. We then used the three replicates in unstimulated conditions and in $4 \mathrm{~h}$ LPS stimulation to perform a differential expression analysis using DESeq $2^{79}$ Wald test, and $P$ values were adjusted for multiple testing by estimating the FDR. A similar procedure was performed with mouse and rat dsRNA stimulation (with $4 \mathrm{~h}$ dsRNA stimulation versus unstimulated conditions).

To quantify transcriptional divergence in immune response between species, we focused on genes that have annotated one-to-one orthologues across the studied species.

We define a measure of response divergence by calculating the differences between the fold-change estimates across the orthologues: response divergence $=\log \left[1 / 3 \times \sum_{j}(\log [\mathrm{FC} \mathrm{pig}]-\log [\mathrm{FC} \text { glire }])^{2}\right]$. For each gene, the fold-change in the outer group (pig), is subtracted from the fold-change in the orthologues of the three glires (mouse, rat and rabbit), and the average of the square values of these subtractions is taken as the response divergence measure. In most of the analyses, we focus on the 2,336 LPS-responsive genes-genes that are differentially expressed in response to LPS (genes that have an FDR-corrected $P<0.01$ in mouse, and have annotated one-to-one orthologues in the other three species).

Promoter elements, gene function and cell-to-cell variability analyses. Promoter elements (TATA and CGIs), gene function and cell-to-cell variability analyses were performed as described in the fibroblasts section. Mouse genes were used as the reference for gene function and TSS annotations. For variability analysis, we used one representative replicate out of three.

Statistical analysis and reproducibility. Statistical analyses were done with R version 3.3.2 for Fisher's exact test, two-sample Kolmogorov-Smirnov test and Mann-Whitney test. Data in boxplots represent the median, first quartile and third quartile with lines extending to the furthest value within 1.5 of the interquartile range (as implemented by the $\mathrm{R}$ function geom_boxplot). Violin plots show the kernel probability density of the data (as implemented by the R function geom_violin)

All cross-species bulk RNA-seq replicates were successful, except for one macaque individual in which the treated sample had a low RNA quality and was removed from the analysis (along with the matching control). All cross-species ChIP-seq replicates were successful. Cross-species scRNA-seq of fibroblasts was performed in two biological replicates. Results throughout the manuscript relate to the second cross-species biological replicate, for which a higher proportion of cells passed technical quality control. Three out of three replicates for each species and condition were successful when preparing single-cell libraries for mononuclear phagocytes, except for two libraries that failed at the emulsion preparation stage. Two out of two replicates of single-cell in situ RNA hybridization assay were performed and both are shown.

Reporting summary. Further information on research design is available in the Nature Research Reporting Summary linked to this paper.
Code availability. Scripts for major analyses are available at https://github.com/ Teichlab/innate_evo.

\section{Data availability}

Sequencing data have been deposited in ArrayExpress with the following accessions: E-MTAB-5918, E-MTAB-5919, E-MTAB-5920, E-MTAB-6754, E-MTAB-6773, E-MTAB-5988, E-MTAB-5989, E-MTAB-6831, E-MTAB-6066, E-MTAB-7032, E-MTAB-7037, E-MTAB-7051 and E-MTAB-7052.

51. Kilpinen, $\mathrm{H}$. et al. Common genetic variation drives molecular heterogeneity in human iPSCs. Nature 546, 370-375 (2017).

52. Schmidl, C., Rendeiro, A. F., Sheffield, N. C. \& Bock, C. ChIPmentation: fast, robust, low-input ChIP-seq for histones and transcription factors. Nat. Methods 12, 963-965 (2015).

53. Picelli, S. et al. Full-length RNA-seq from single cells using Smart-seq2. Nat. Protocols 9, 171-181 (2014).

54. Patro, R., Duggal, G., Love, M. I., Irizarry, R. A. \& Kingsford, C. Salmon provides fast and bias-aware quantification of transcript expression. Nat. Methods $\mathbf{1 4}$, 417-419 (2017)

55. Robinson, M. D., McCarthy, D. J. \& Smyth, G. K. edgeR: a Bioconductor package for differential expression analysis of digital gene expression data. Bioinformatics 26, 139-140 (2010).

56. Nourmohammad, A. et al. Adaptive evolution of gene expression in Drosophila. Cell Reports 20, 1385-1395 (2017).

57. Zhang, H. M. et al. AnimalTFDB: a comprehensive animal transcription factor database. Nucleic Acids Res. 40, D144-D149 (2012).

58. Binns, D. et al. QuickGO: a web-based tool for Gene Ontology searching. Bioinformatics 25, 3045-3046 (2009).

59. Kent, W. J. et al. The human genome browser at UCSC. Genome Res. 12, 996-1006 (2002)

60. Langmead, B. \& Salzberg, S. L. Fast gapped-read alignment with Bowtie 2. Nat. Methods 9, 357-359 (2012).

61. Li, H. et al. The sequence alignment/map format and SAMtools. Bioinformatics 25, 2078-2079 (2009).

62. Zhang, Y. et al. Model-based analysis of ChIP-Seq (MACS). Genome Biol. 9, R137 (2008).

63. Quinlan, A. R. \& Hall, I. M. BEDTools: a flexible suite of utilities for comparing genomic features. Bioinformatics 26, 841-842 (2010).

64. Kuhn, R. M. et al. The UCSC genome browser database: update 2007. Nucleic Acids Res. 35, D668-D673 (2007).

65. Mathelier, A. et al. JASPAR 2016: a major expansion and update of the open-access database of transcription factor binding profiles. Nucleic Acids Res. 44, D110-D115 (2016).

66. Grant, C. E., Bailey, T. L. \& Noble, W. S. FIMO: scanning for occurrences of a given motif. Bioinformatics 27, 1017-1018 (2011).

67. Pollard, K. S., Hubisz, M. J., Rosenbloom, K. R. \& Siepel, A. Detection of nonneutral substitution rates on mammalian phylogenies. Genome Res. 20 , 110-121 (2010).

68. Kolodziejczyk, A. A. et al. Single cell RNA-sequencing of pluripotent states unlocks modular transcriptional variation. Cell Stem Cell 17, 471-485 (2015).

69. Vallejos, C. A., Marioni, J. C. \& Richardson, S. BASiCS: Bayesian analysis of single-cell sequencing data. PLOS Comput. Biol. 11, e1004333 (2015).

70. Martinez-Jimenez, C. P. et al. Aging increases cell-to-cell transcriptional variability upon immune stimulation. Science 355, 1433-1436 (2017)

71. Smoot, M. E., Ono, K., Ruscheinski, J., Wang, P. L. \& Ideker, T. Cytoscape 2.8: new features for data integration and network visualization. Bioinformatics 27, 431-432 (2011)

72. Lindblad-Toh, K. et al. A high-resolution map of human evolutionary constraint using 29 mammals. Nature 478, 476-482 (2011).

73. Herrero, J. et al. Ensembl comparative genomics resources. Database 2016, bav096 (2016).

74. De Bie, T., Cristianini, N., Demuth, J. P. \& Hahn, M. W. CAFE: a computational tool for the study of gene family evolution. Bioinformatics 22, 1269-1271 (2006).

75. Capra, J. A., Williams, A. G. \& Pollard, K. S. ProteinHistorian: tools for the comparative analysis of eukaryote protein origin. PLOS Comput. Biol. $\mathbf{8}$, e1002567 (2012).

76. Szklarczyk, D. et al. STRING v10: protein-protein interaction networks, integrated over the tree of life. Nucleic Acids Res. 43, D447-D452 (2015).

77. Zheng, G. X. et al. Massively parallel digital transcriptional profiling of single cells. Nat. Commun. 8, 14049 (2017).

78. Satija, R., Farrell, J. A., Gennert, D., Schier, A. F. \& Regev, A. Spatial reconstruction of single-cell gene expression data. Nat. Biotechnol. 33, 495-502 (2015).

79. Love, M. I., Huber, W. \& Anders, S. Moderated estimation of fold-change and dispersion for RNA-seq data with DESeq2. Genome Biol. 15, 550 (2014). 

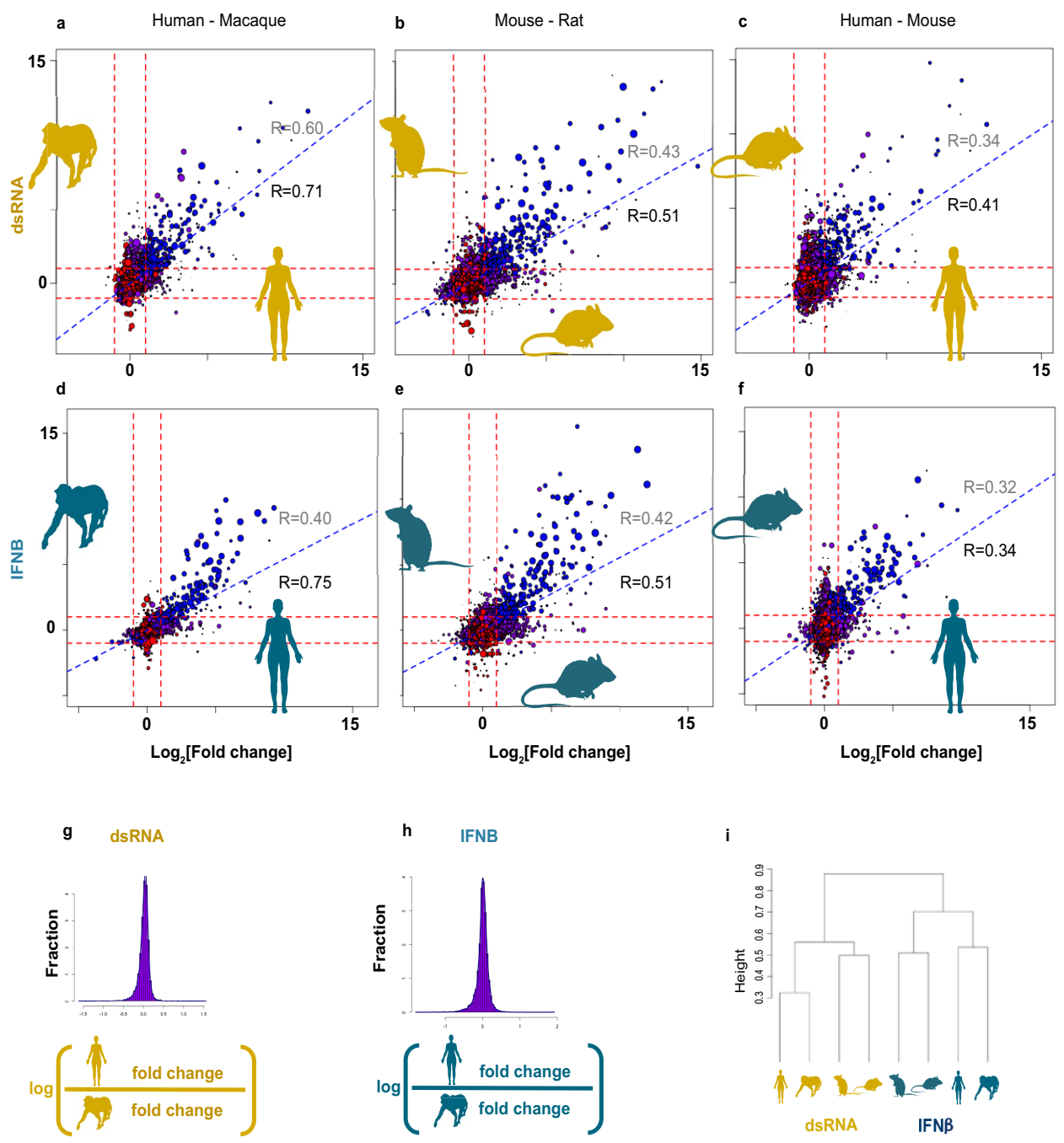

Extended Data Fig. 1 | Fibroblast response to dsRNA and IFNB across species. To study the similarity in response to treatment across species, we plotted the fold-change values of all expressed genes (with one-to-one orthologues) between pairs of species (human-macaque, mouse-rat and human-mouse) in response to dsRNA (poly(I:C)) $(\mathbf{a}-\mathbf{c})$. As a control, we performed the same procedure with IFNB stimulations $(\mathbf{d}-\mathbf{f})$. Foldchanges were inferred from differential expression analyses, determined by the exact test in the edgeR package ${ }^{6}$ and based on $n=6,5,3$ and 3 individuals from human, macaque, rat and mouse, respectively. Spearman correlations between all expressed one-to-one orthologues are shown in grey, Spearman correlations between the subset of differentially expressed genes (FDR-corrected $P<0.01$ in at least one species) appear in black. Number of genes shown is $n=11,035,11,005,11,137,10,851,10,826$ and 10,957 in a-f, respectively. Genes are coloured blue if they were differentially expressed (FDR-corrected $P<0.01$ ) in both species, purple if they were differentially expressed in only one species, or red if they were not differentially expressed. $\mathbf{g}, \mathbf{h}$, Density plots of ratio of fold-change in response to dsRNA or to IFNB. g, Comparison between human and macaque orthologues in dsRNA response. $\mathbf{h}$, Comparison between human and mouse orthologues in IFNB response. i, Dendrogram based on the fold-change in response to dsRNA or to IFNB across 9,835 one-to-one orthologues in human, macaque, rat and mouse. 

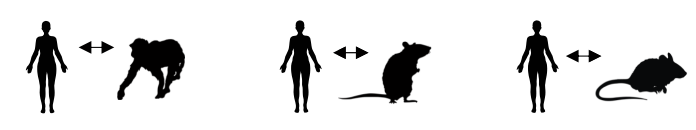

Conserved
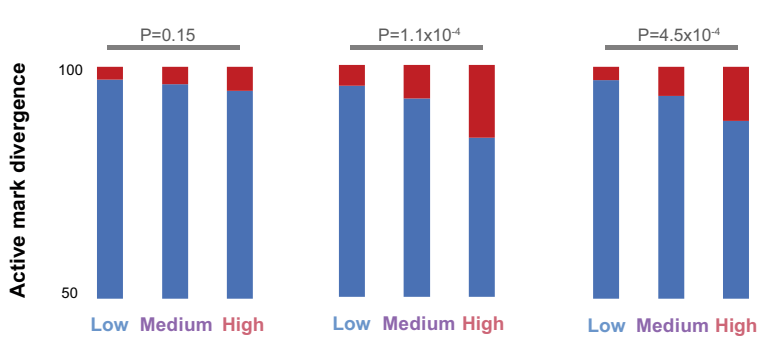

b
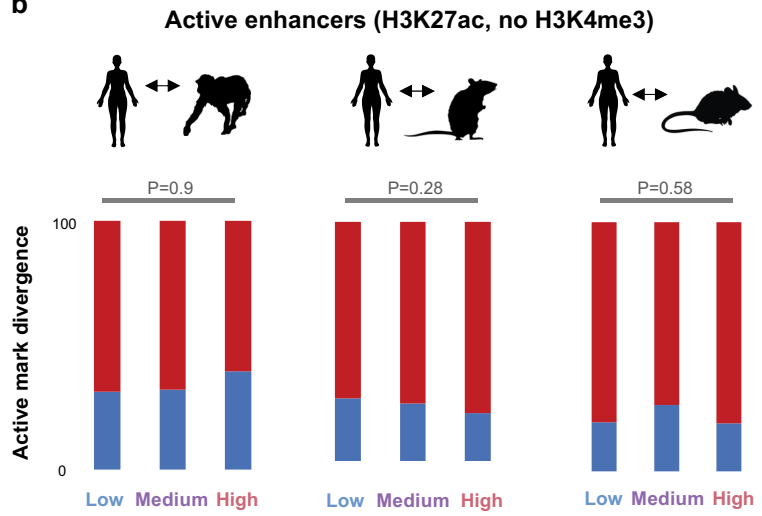

Extended Data Fig. 2 | Correspondence of transcriptional divergence and divergence of active promoters and enhancers. Comparison of divergence in transcriptional response to dsRNA with divergence of active chromatin marks in active promoters (a, profiled using H3K4me3 in proximity to gene's TSS) and enhancers (b, H3K27ac without overlapping $\mathrm{H} 3 \mathrm{~K} 4 \mathrm{me} 3)$. Chromatin marks were linked to genes on the basis of their proximity to the gene's TSS. Chromatin marks were obtained from $n=3$ individuals in each of the four species, from fibroblasts stimulated with dsRNA or left untreated. The statistics are based on $n=855,818$ and 813 human genes that have a linked $\mathrm{H} 3 \mathrm{~K} 4 \mathrm{me} 3$ mark with a syntenic region in macaque, rat and mouse, respectively (a); and on $n=326,241$ and 242 human genes that have a linked H3K27ac mark with a syntenic region in macaque, rat and mouse, respectively (b). Each panel shows the fraction of conserved marks between human and macaque, rat or mouse, in genes that

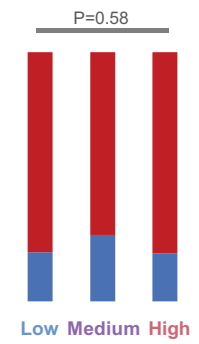

have high, medium and low divergence in their transcriptional response. In each column, the histone mark's signal was compared between human and the syntenic region in one of the three other species. If an active mark was found in the corresponding syntenic region, the linked gene was considered to have a conserved active mark (promoter or enhancer). The fractions of genes with conserved promoters (or enhancers) in each pair of species were compared between high- and low-divergence genes using a one-sided Fisher's exact test. When comparing active promoter regions of high-versus low-divergence genes, we observe that low-divergence genes have a significantly higher fraction of conserved marks in rodents. This suggests an agreement between divergence at the transcriptional and chromatin levels in active promoter regions. In active enhancer regions, we do not observe these patterns, suggesting that the major contribution to divergence comes from promoters. 


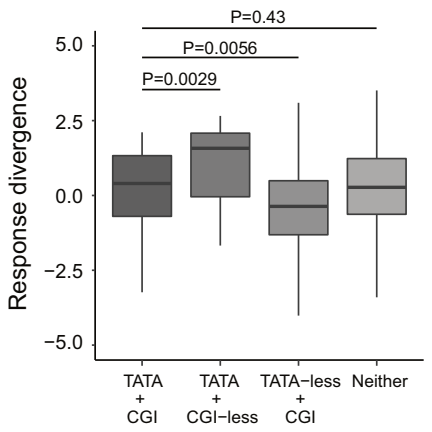

Extended Data Fig. 3 | Comparison of response divergence of genes containing various promoter elements. Comparison of response divergence between genes with and without a TATA-box and a CGI. Left, fibroblasts $(n=14,14,633$ and 294 differentially expressed genes with only TATA-box element, with both CGI and TATA-box elements, with only CGI, and with neither element in their promoters, respectively); right, phagocytes $(n=13,29,1,718$ and 576 differentially expressed genes with only a TATA-box element, with both CGI and TATA-box elements, with

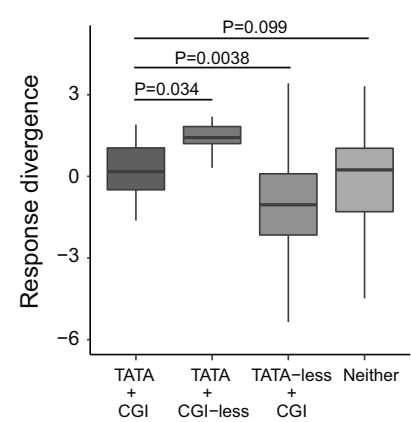

only a CGI, and with neither element in their promoters, respectively). Genes with a TATA-box without a CGI have higher response divergence than genes with both elements. Genes with a CGI but without a TATAbox diverge more slowly than genes with both elements. Genes with both elements do not differ significantly in their divergence from genes lacking both elements (one-sided Mann-Whitney test). Data in boxplots represent the median, first quartile and third quartile with lines extending to the furthest value within 1.5 of the IQR. 


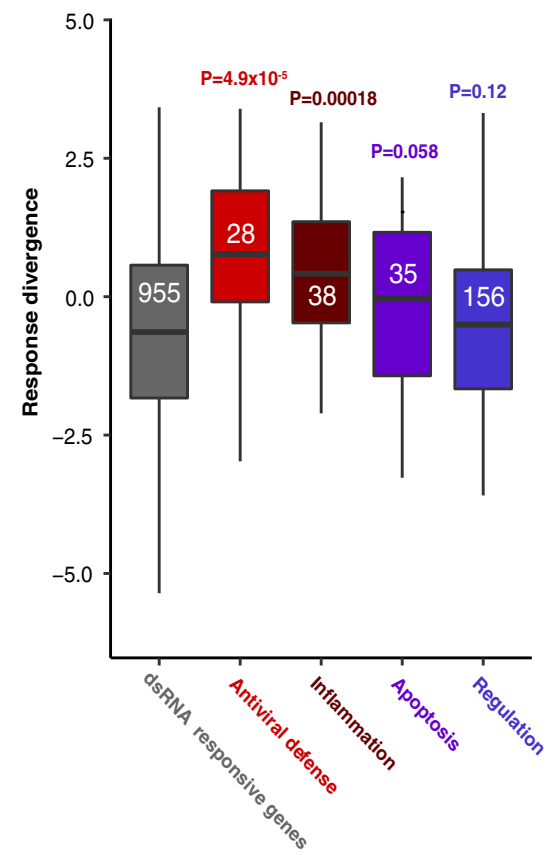

Extended Data Fig. 4 | Response divergence of molecular processes upregulated in immune response. Left, distributions of divergence values of $n=955$ dsRNA-responsive genes in fibroblasts and subsets of this group belonging to different biological processes. For each functional subset, the distribution of divergence values is compared with the set of 955 dsRNAresponsive genes using a one-sided Mann-Whitney test. FDR-corrected $P$ values are shown above each group and group size is shown inside each box. Right, distributions of divergence values of $n=2,336$ LPS-responsive

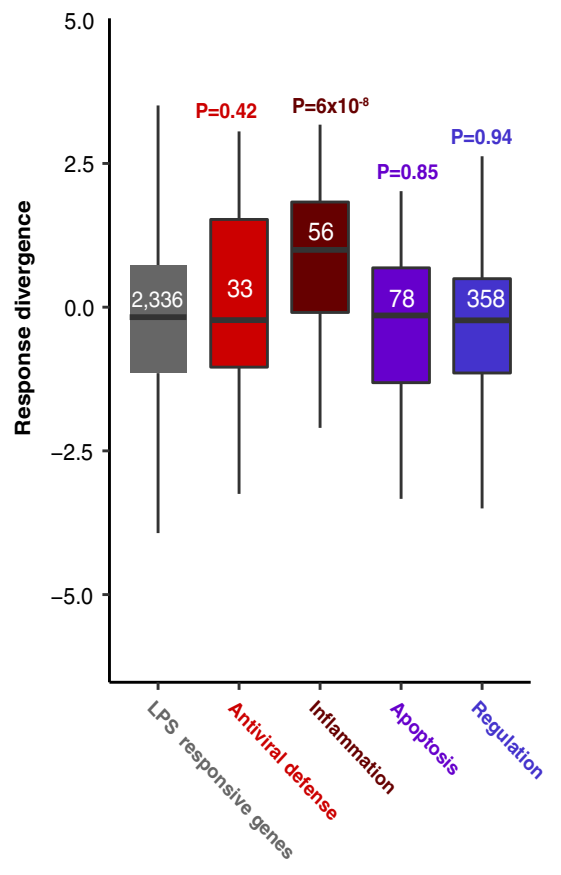

genes in mononuclear phagocytes and subsets of this group belonging to different biological processes. For each functional subset, the distribution of divergence values is compared with the set of 2,336 LPS-responsive genes. FDR-corrected $P$ values (one-sided Mann-Whitney test) are shown above each group and group size is shown inside each box. Data in boxplots represent the median, first quartile and third quartile with lines extending to the furthest value within 1.5 of the IQR. 

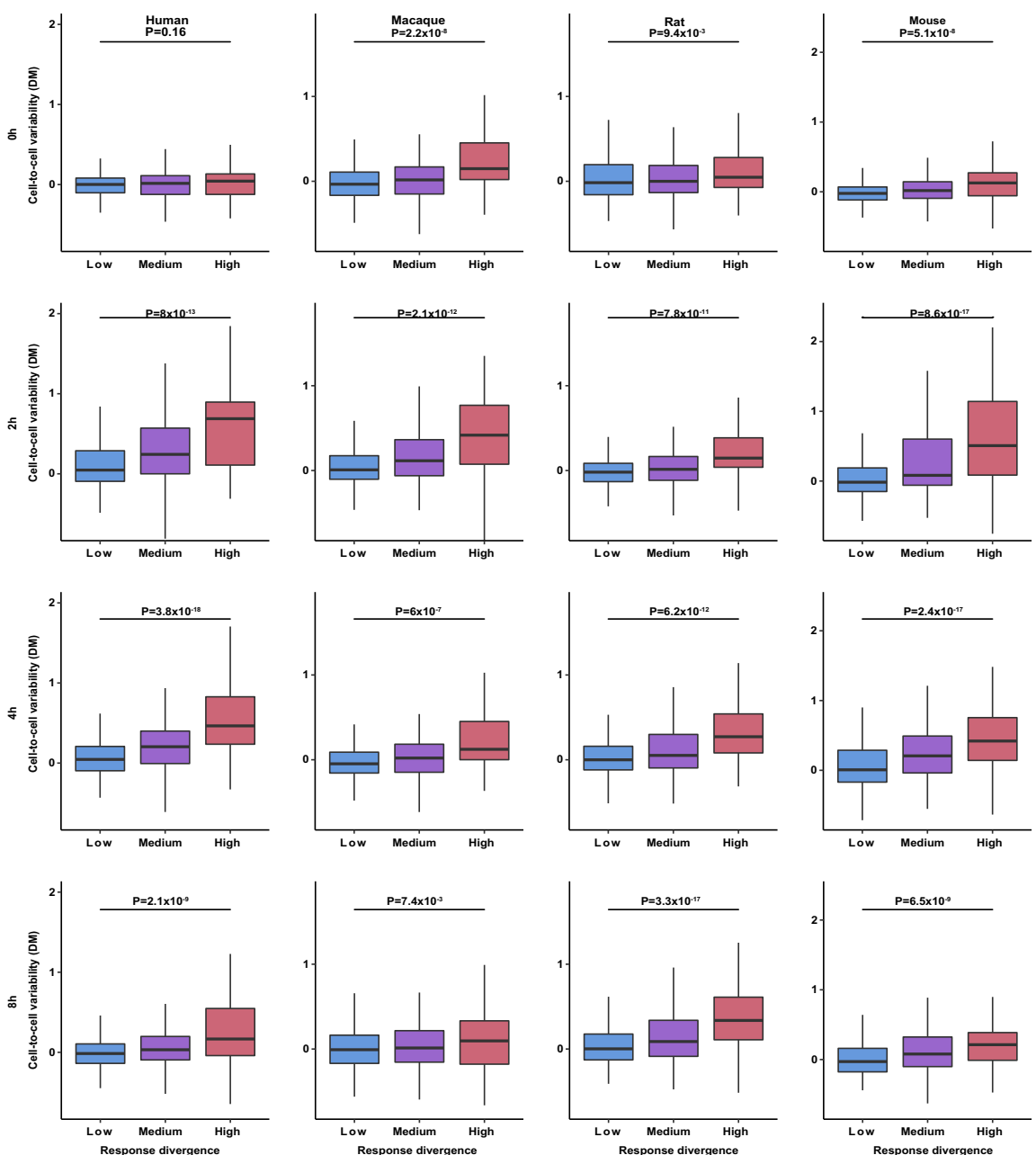

Extended Data Fig. 5 | Cell-to-cell variability versus response divergence across species and conditions in fibroblasts after dsRNA stimulation. Cell-to-cell variability values, as measured with DM across individual cells, compared with response divergence between species (grouped into low, medium and high divergence). Variability values are based on $n=29,56,55,35$ human cells, $n=20,32,29,13$ rhesus cells, $n=33,70,65,40$ rat cells, and $n=53,81,59,30$ mouse cells, stimulated

with dsRNA for $0,2,4$ and $8 \mathrm{~h}$, respectively. Rows represent different dsRNA stimulation time points $(0,2,4$ and $8 \mathrm{~h})$, and columns represent different species as shown. High-divergence genes were compared with low-divergence genes using a one-sided Mann-Whitney test. Data in boxplots represent the median, first quartile and third quartile with lines extending to the furthest value within 1.5 of the IQR. 

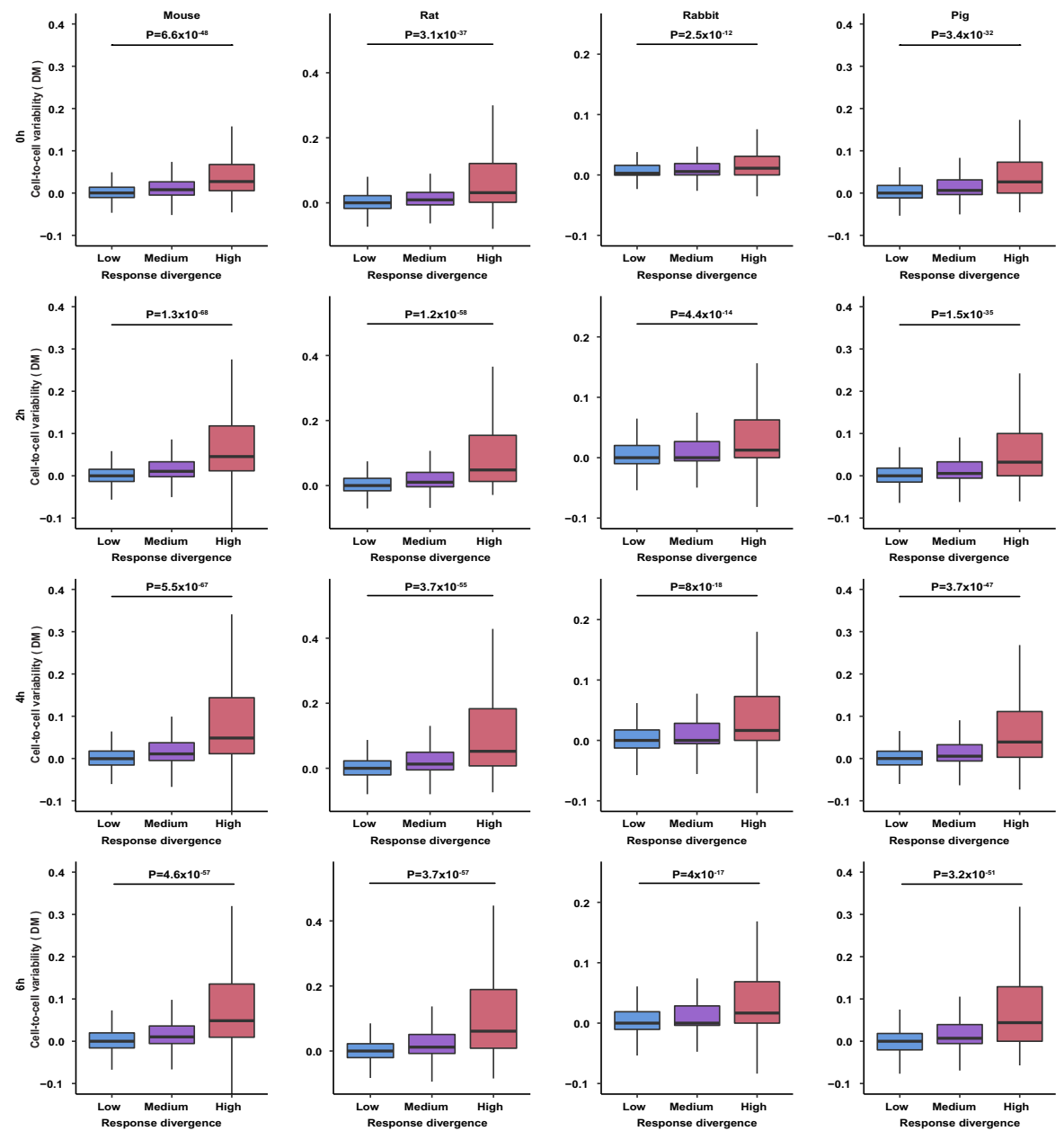

Extended Data Fig. 6 | Cell-to-cell variability versus response divergence across species and conditions in mononuclear phagocytes after LPS stimulation. Cell-to-cell variability values, as measured with DM across cells, compared with response divergence between species (grouped into low, medium and high divergence). Variability values are based on $n=3,519,4,321,3,293,2,126$ mouse cells, $n=2,266,2,839,1,963$, 1,607 rat cells, $n=3,275,1,820,1,522,1,660$ rabbit cells, and $n=1,748$,

$1,614,1,899,1,381$ pig cells, stimulated with LPS for $0,2,4$ and $6 \mathrm{~h}$, respectively. Rows represent different LPS stimulation time points $(0,2,4$ and $6 \mathrm{~h})$, and columns represent different species as shown.

High-divergence genes were compared with low-divergence genes using a one-sided Mann-Whitney test. Data in boxplots represent the median, first quartile and third quartile with lines extending to the furthest value within 1.5 of the IQR. 

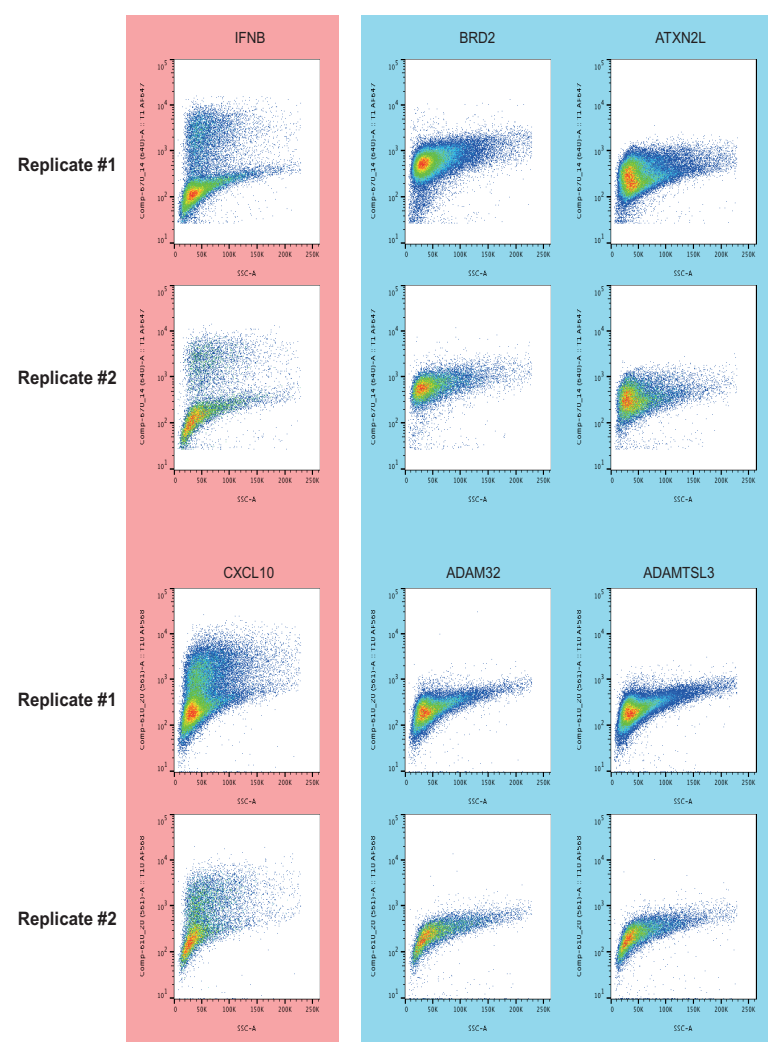
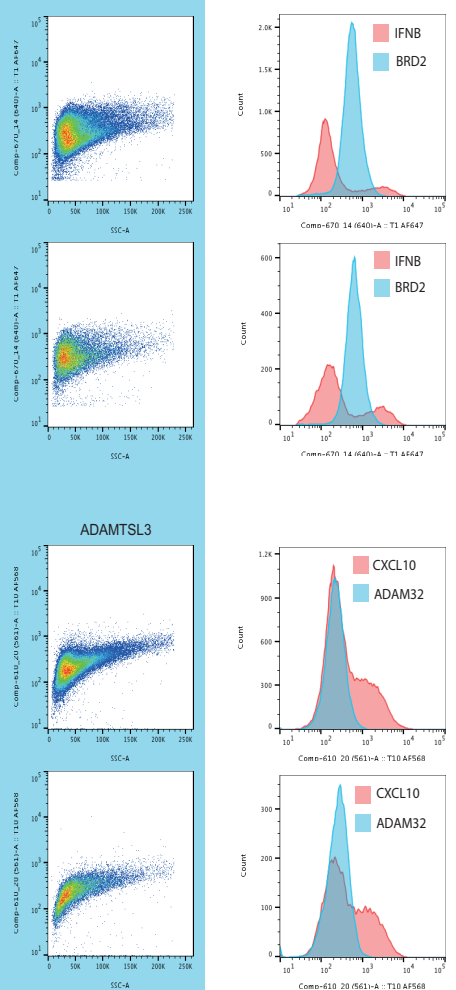

Extended Data Fig. 7 | Cell-to-cell variability of cytokine expression in single cell in situ RNA hybridization assay combined with flow cytometry (PrimeFlow). PrimeFlow measurement of two cytokine genes (IFNB and CXCL10) that show high cell-to-cell variability in scRNAseq. As controls, two genes matched on expression levels (ATXN2L and $A D A M 32$ ) but that show low cell-to-cell variability in scRNA-seq data are shown. As the expression of cytokines is at the low end of the distribution, we also chose two genes with middle-range expression values (ADAMTSL3 and BRD2) as additional controls. The experiment was performed in $n=2$ independent replicates, originating from the same individual. Both replicates are shown. a, Pseudocolour contour plot for RNA target expression in dsRNA-stimulated human fibroblasts. The $x$-axis shows area of side scatter (SSC-A) and the $y$-axis shows fluorescent signal for target RNA probes. RNA targets detected by the same fluorescent channel are displayed together. Top, IFNB and control genes BRD2 and $A T X N 2 L$, type 1 probe, Alexa FluorTM 647. Bottom, CXCL10 and control genes ADAMTSL3 and ADAM32, type 10 probe, Alexa FluorTM 568. The cytokine genes display a broader range of fluorescence signal than the controls. b, Histograms comparing fluorescence of cytokine and control pairs (IFNB-BRD2 for type 1 probe and CXCL10-ADAM32 for type 10 probe). The histograms show a bimodal distribution of expression signal for the two cytokine genes (IFNB and CXCL10, red), but not for controls (blue). This agrees with scRNA-seq data in which CXCL10 and IFNB display high levels of cell-to-cell variability. 


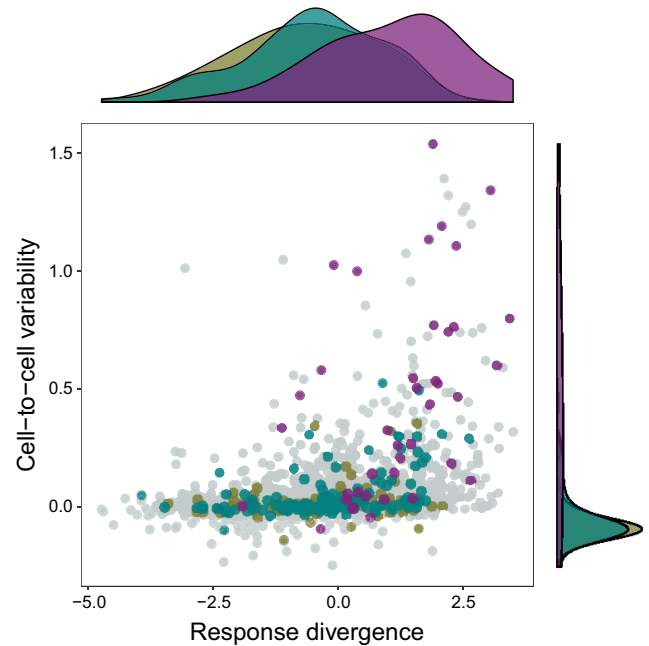

Extended Data Fig. 8 | Cell-to-cell variability levels and response divergence of cytokines, transcription factors and kinases in response to LPS stimulation of phagocytes. A scatter plot showing divergence in response to LPS across species and transcriptional cell-to-cell variability in mouse mononuclear phagocytes following $4 \mathrm{~h}$ of LPS treatment, in $n=2,262$ LPS-responsive genes. Purple, cytokines; green, transcription factors; beige, kinases. The distributions of divergence values and cellto-cell variability values of each of the three functional groups are shown above and to the right of the scatter plot, respectively. 

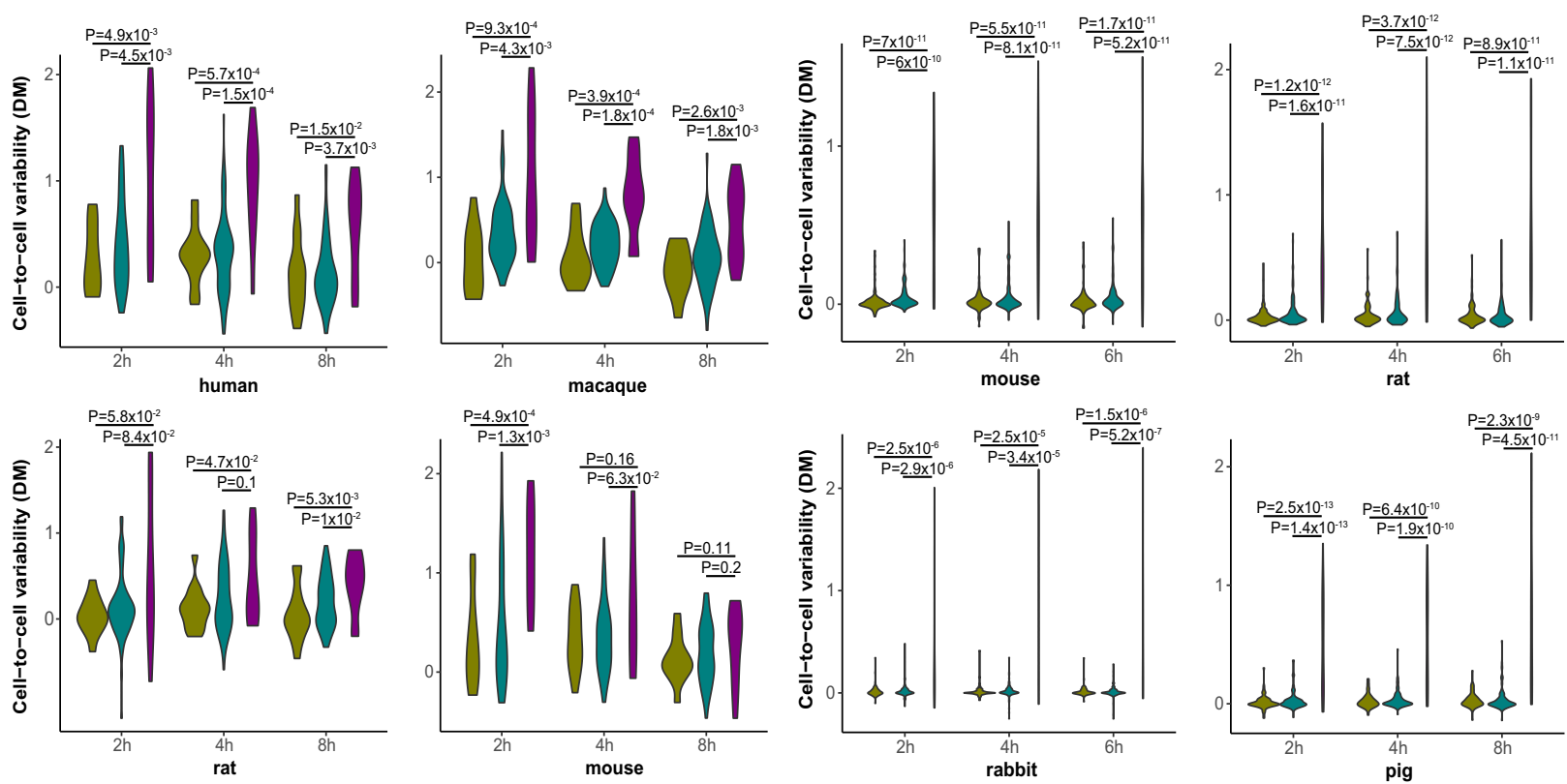

Extended Data Fig. 9 | Cell-to-cell variability levels in cytokines, transcription factors and kinases across species and stimulation time points. Violin plots showing the distribution of cell-to-cell variability values (DM) of cytokines, transcription factors and kinases during immune stimulation. Left, fibroblast dsRNA stimulation time course. Number of cells used in each species (at 2, 4, $8 \mathrm{~h}$ dsRNA, respectively): human, 56, 55, 35; macaque, 32, 29, 13; rat, 70, 65, 40; mouse, 81, 59, 30.

Right, phagocyte LPS stimulation time course. Number of cells used in each species (at 2, 4, $6 \mathrm{~h}$ LPS, respectively): mouse, 4,321, 3,293, 2,126; rat, $2,839,1,963,1,607$; rabbit, $1,820,1,522,1,660$; pig, 1,614, 1,899, 1,381. For both panels, colours as in Fig. 3c. Comparisons between groups of genes were performed using one-sided Mann-Whitney tests. Violin plots show the kernel probability density of the data. 
RESEARCH ARTICLE

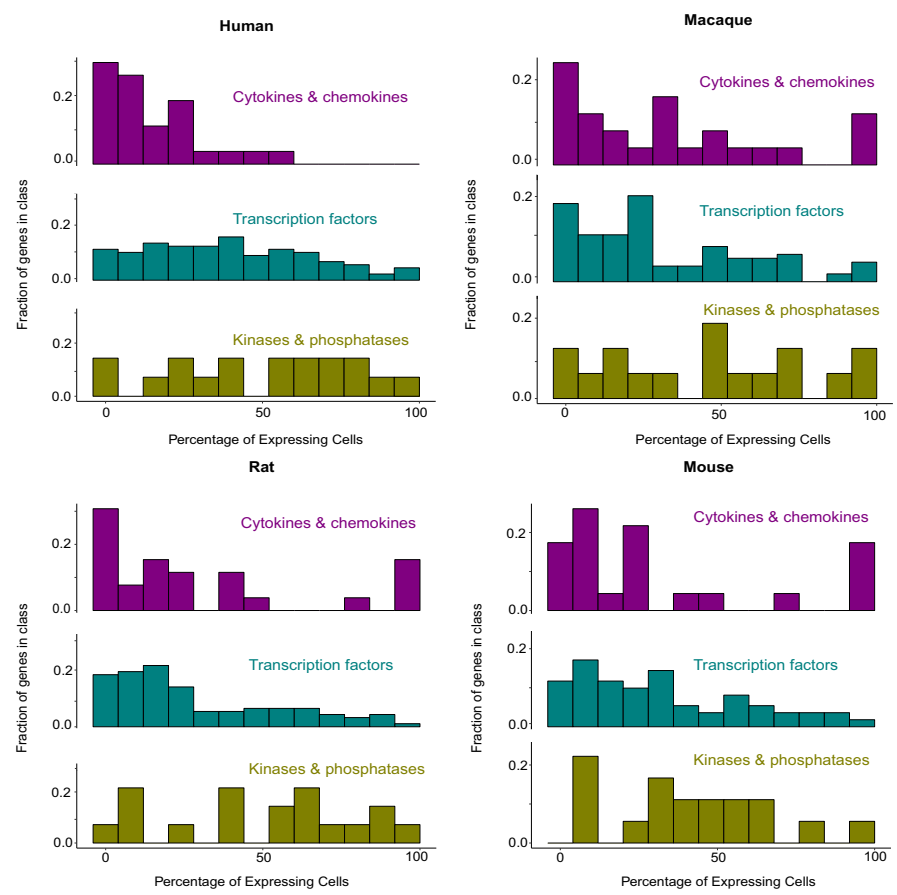

Extended Data Fig. 10 | Percentage of cells expressing cytokines, transcription factors and kinase. Histograms showing the percentage of fibroblasts expressing cytokines (top), transcription factors (middle) and kinase (bottom) following $4 \mathrm{~h}$ dsRNA stimulation, in human, macaque, rat and mouse cells (based on $n=55,29,65$ and 59 cells, respectively).
The percentage of expressing cells is divided into 13 bins ( $x$-axis). The $y$-axis represents the fraction of genes from this gene class (for example, cytokines) that are expressed in each bin (for example, in human, nearly $30 \%$ of the cytokine genes ( $y$-axis) are expressed in the first bin, corresponding to expression in fewer than $8 \%$ of cells).

(C) 2018 Springer Nature Limited. All rights reserved. 


\section{Reporting Summary}

Nature Research wishes to improve the reproducibility of the work that we publish. This form provides structure for consistency and transparency in reporting. For further information on Nature Research policies, see Authors \& Referees and the Editorial Policy Checklist.

\section{Statistical parameters}

When statistical analyses are reported, confirm that the following items are present in the relevant location (e.g. figure legend, table legend, main text, or Methods section).

$\mathrm{n} / \mathrm{a} \mid$ Confirmed

$\bigotimes$ The exact sample size $(n)$ for each experimental group/condition, given as a discrete number and unit of measurement

$\square$ An indication of whether measurements were taken from distinct samples or whether the same sample was measured repeatedly

The statistical test(s) used AND whether they are one- or two-sided

Only common tests should be described solely by name; describe more complex techniques in the Methods section.

Х $\square$ A description of all covariates tested

$\bigotimes$ A description of any assumptions or corrections, such as tests of normality and adjustment for multiple comparisons

$\square$ A full description of the statistics including central tendency (e.g. means) or other basic estimates (e.g. regression coefficient) AND

variation (e.g. standard deviation) or associated estimates of uncertainty (e.g. confidence intervals)

$\varnothing$ For null hypothesis testing, the test statistic (e.g. $F, t, r$ ) with confidence intervals, effect sizes, degrees of freedom and $P$ value noted

$\triangle$ Give $P$ values as exact values whenever suitable.

Х $\square$ For Bayesian analysis, information on the choice of priors and Markov chain Monte Carlo settings

Х $\square$ For hierarchical and complex designs, identification of the appropriate level for tests and full reporting of outcomes

$\square$ Estimates of effect sizes (e.g. Cohen's d, Pearson's $r$ ), indicating how they were calculated

$\varnothing$ Clearly defined error bars

State explicitly what error bars represent (e.g. SD, SE, CI)

Our web collection on statistics for biologists may be useful.

\section{Software and code}

Policy information about availability of computer code

Data collection Software used include: edgeR, Salmon, Trim Galore, DESeq2, Bowtie2, MACS2, liftOver, FIMO, BASiCS, 10X Genomics' Cell Ranger, Seurat, as well as R version 3.3.2 (2016-10-31) and Perl to run many of the mentioned programs. Detailed parameters of each of the programs are mentioned in relevant sections in Methods.

Data analysis

Software used include: edgeR, Salmon, Trim Galore, DESeq2, Bowtie2, MACS2, liftOver, FIMO, BASiCS, 10X Genomics' Cell Ranger, Seurat, as well as R version 3.3.2 (2016-10-31) and Perl to run many of the mentioned programs. Detailed parameters of each of the programs are mentioned in relevant sections in Methods.

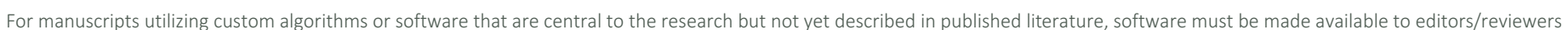

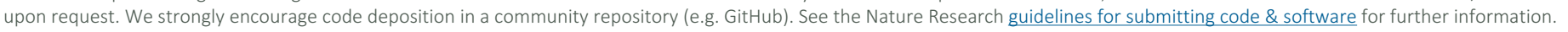


Policy information about availability of data

All manuscripts must include a data availability statement. This statement should provide the following information, where applicable:

- Accession codes, unique identifiers, or web links for publicly available datasets

- A list of figures that have associated raw data

- A description of any restrictions on data availability

Sequencing data are deposited in ArrayExpress with the following accessions: E-MTAB-5918, E-MTAB-5919, E-MTAB-5920, E-MTAB-6754, E-MTAB-6773, EMTAB-5988, E-MTAB-5989, E-MTAB-6831, E-MTAB-6066, E-MTAB-7032, E-MTAB-7037, E-MTAB-7051, E-MTAB-7052.

\section{Field-specific reporting}

Please select the best fit for your research. If you are not sure, read the appropriate sections before making your selection.

$\bigotimes$ Life sciences $\quad \square$ Behavioural \& social sciences $\quad \square$ Ecological, evolutionary \& environmental sciences

For a reference copy of the document with all sections, see nature.com/authors/policies/ReportingSummary-flat.pdf

\section{Life sciences study design}

All studies must disclose on these points even when the disclosure is negative.

Sample size No statistical methods were used to predetermine sample size. We followed ENCODE guidelines and standards in the field.

Data exclusions Low quality samples were removed (i.e. - cells with low number of mapped reads; bulk RNA samples with RIN<9; batch with low fraction of high quality cells) - exclusion criteria for each case are comprehensively detailed in the relevant Methods section.

Replication All cross-species bulk RNA-Seq replicates were successful, except for one macaque individual where the treated sample had a low RNA quality and was removed from the analysis (along with the matching control). All cross-species ChIP-Seq replicates were successful. Cross-species scRNA-seq of fibroblasts was performed in two biological replicates. Results throughout the manuscript relate to the second cross-species biological replicate where a higher proportion of cells passed technical QC. Three out of three replicates for each species and condition were successful when preparing single-cell libraries for mononuclear phagocytes, except for two libraries that failed at the emulsion preparation stage. Two out of two replicates of single-cell in situ RNA hybridization assay were performed and both are shown.

Randomization Individuals from each species were randomly allocated to each cross-species experimental batch.

Blinding Systematic blinding was not performed. It was deemed unnecessary for this study since all samples were processed in parallel.

\section{Reporting for specific materials, systems and methods}

Materials \& experimental systems

$\mathrm{n} / \mathrm{a}$ Involved in the study

$\triangle \square$ Unique biological materials

$\square$ Antibodies

Х Eukaryotic cell lines

Х Palaeontology

$\square \searrow$ Animals and other organisms

$\square$ \ Human research participants

\begin{tabular}{l} 
Methods \\
\hline n/a Involved in the study \\
$\square$ ChIP-seq \\
$\square$ Flow cytometry
\end{tabular}

\section{Antibodies}

Antibodies used

Validation
Antibodies used for ChiP-seq :

Diagenode, C15410003-50, H3K4me3 polyclonal antibody - Premium

Diagenode, C15410196, H3K27ac polyclonal antibody - Premium

Antibodies were purchased from Diagenode. The antibodies have been validated by the company using dot plot, western blot, immunofluorescence, ChIP-qPCR on known loci and full-scale ChIP-seq experiments - see information regarding H3K4me3 antibody here: https://www.diagenode.com/en/p/h3k4me3-polyclonal-antibody-premium-50-ug-50-ul. The validation 


\section{Animals and other organisms}

Policy information about studies involving animals; ARRIVE guidelines recommended for reporting animal research

Laboratory animals

Wild animals

Field-collected samples
No experiments were performed using lab animals. Dermal fibroblast cells were isolated from sexually-mature female individuals including rhesus macaques ( 12 year old), C57BL/6 mice ( 8 week old) and brown Norway rats ( 8 week old). Bone marrow-derived phagocytes were derived from 8-week old female individuals from C57BL/6 mouse, brown Norway rat, and rabbit and from 2-3 week old female pigs.

No wild animals were used.

No samples were field-collected.

\section{Human research participants}

Policy information about studies involving human research participants

Population characteristics

Human samples for cross-species comparisons were derived from healthy adult females of European ancestry from the Hipsci consortium, with the age range of 40-49. For the large human cohort, we used samples derived from healthy adults ( 28 males and 42 and females) of European ancestry from the Hipsci consortium, with the age range of 30-79 (see details for each individual in E-MTAB-7032).

Recruitment

Human cells were obtained from the Hipsci project, where they were collected from consented research volunteers recruited from the NIHR Cambridge BioResource. There was no self-selection bias involved.

\section{ChIP-seq}

Data deposition

Х Confirm that both raw and final processed data have been deposited in a public database such as GEO.

\Confirm that you have deposited or provided access to graph files (e.g. BED files) for the called peaks.

Data access links

May remain private before publication.

Files in database submission
Data is uploaded to ArrayExpress:

Experiment: E-MTAB-5918

104 files - 2 fastq files for each of the 52 samples, including three biological replicates from each of the species and each condition, for H3K27ac and for H3K4me3:

HS3_H3K4me3_UNST

HS3_H3K27ac_UNST

RH3_H3K4me3_UNST

RH3_H3K27ac_UNST

BR3 H3K4me3 UNST

BR3_H3K27ac_UNST

MM3_H3K4me3_UNST

MM3_H3K27ac_UNST

HS3_H3K4me3_PIC4

HS3_H3K27ac_PIC4

RH3_H3K4me3_PIC4

RH3_H3K27ac_PIC4

BR3_H3K4me3_PIC4

BR3 H3K27ac PIC4

MM3_H3K4me3_PIC4

MM3_H3K27ac_PIC4

HS4_H3K4me3_UNST

HS4 H3K27ac UNST

RH4_H3K4me3_UNST

$\mathrm{RH} 4$ H3K27ac UNST

BR4_H3K4me3_UNST

BR4_H3K27ac_UNST

MM4_H3K4me3_UNST

MM4_H3K27ac_UNST

HS4_H3K4me3_PIC4

HS4_H3K27ac_PIC4

RH4_H3K4me3_PIC4

RH4_H3K27ac_PIC4

BR4_H3K4me3_PIC4

BR4_H3K27ac_PIC4

MM4_H3K4mé3_PIC4

MM4_H3K27ac_PIC4 
HS5_H3K4me3_UNST

HS5 H3K27ac UNST

RH5_H3K4me3_UNST

RH5 H3K27ac UNST

BR5_H3K4me3_UNST

BR5_H3K27ac UNST

MM5_H3K4me3_UNST

MM5_H3K27ac_UNST

HS5 H3K4me3 PIC4

HS5_H3K27ac_PIC4

RH5 H3K4me3 PIC4

RH5_H3K27ac_PIC4

BR5 H3K4me3 PIC4

BR5_H3K27ac_PIC4

MM5 H3K4me3 PIC4

MM5_H3K27ac_PIC4

HS5 CTRL

RH5_CTRL

BR5 CTRL

MM5_CTRL

Genome browser session

(e.g. UCSC)

\section{Methodology}

Replicates

Sequencing depth

Antibodies

Peak calling parameters

Data quality

Software
A link to the UCSC genome browser session is available at:

http://ec2-34-223-252-194.us-west-2.compute.amazonaws.com:8001/\#/base/experiment/5/chromatin

3 biological replicates for each condition (control and dsRNA treatment) and each of the 4 species (human, macaque, mouse and rat); ChIP-seq data of two histone marks - H3K4me3 and H3K27ac.

On average, 10 million pair-end reads for each experiment

Diagenode, C15410003-50, H3K4me3 polyclonal antibody - Premium

Diagenode, C15410196, H3K27ac polyclonal antibody - Premium

macs2 callpeak function with "--nomodel -f BAMPE -B --SPMR -g xx" flags, see Methods section in the manuscript for the values of "xx".

(A) Number of peaks with $q<0.01$ in each of the libraries is $>25,000$. (B) Visual inspection of signal on UCSC genome browser. These can be observed for all samples in our website: https://teichlab.sanger.ac.uk/\#/base/experiment/5/ chromatin

MACS2, FIMO, Bowtie2, and visualization using UCSC genome browser, as detailed in Methods.

\section{Flow Cytometry}

Plots

Confirm that:

Х The axis labels state the marker and fluorochrome used (e.g. CD4-FITC).

Х The axis scales are clearly visible. Include numbers along axes only for bottom left plot of group (a 'group' is an analysis of identical markers).

\All plots are contour plots with outliers or pseudocolor plots.

Х A numerical value for number of cells or percentage (with statistics) is provided.

\section{Methodology}

Sample preparation

Instrument

\section{Software}

Cell population abundance

Gating strategy

Tick this box to confirm that a figure exemplifying the gating strategy is provided in the Supplementary Information. target mRNAs.
Human fibroblasts were treated with poly I:C for 4 hrs to induce innate immune response. Cells were harvested and centrifuged, and subject to the The PrimeFlowTM RNA assay (Affymetrix eBioscience) according to the manufacturer's protocol to stain for

\section{BD LSRFortessa}

BD FACS software was used for collection and FlowJo v10.3 was used for analysis.

\section{$10^{\wedge} 4-10^{\wedge} 5$ cells per sample were obtained.}

No gating was performed; only the debris was removed based on very low FSC and SSC. 